\title{
Engineered cell differentiation and sexual reproduction in probiotic and mating yeasts
}

\section{Short title}

Engineering mating activation in yeasts.

\section{One-sentence summary}

Enabling sensing in probiotic yeast and synthetic yeast mating with human and fungal $\mathrm{G}$ protein-coupled receptors.

Emil D. Jensen ${ }^{1, \star}$, Marcus Deichmann ${ }^{1}$, Xin $\mathrm{Ma}^{1}$, Rikke U. Vilandt ${ }^{1}$, Giovanni Schiesaro ${ }^{1}$, Marie B. Rojek ${ }^{1}$, Bettina Lengger ${ }^{1}$, Line Eliasson ${ }^{1}$, Justin M. Vento ${ }^{2}$, Deniz Durmusoglu ${ }^{2}$, Sandie P. Hovmand ${ }^{1}$, Ibrahim Al'Abri ${ }^{2}$, Jie Zhang ${ }^{1}$, Nathan Crook $^{2}$, and Michael K. Jensen ${ }^{1, *}$

${ }^{1}$ Novo Nordisk Foundation Center for Biosustainability, Technical University of Denmark, Kgs. Lyngby, Denmark.

2 Department of Chemical and Biomolecular Engineering, North Carolina State University, Raleigh, North Carolina 27695, United States.

* To whom correspondence should be addressed. Michael K. Jensen, E-mail: mije@biosustain.dtu.dk, and Emil D.Jensen, E-mail: emdaje@biosustain.dtu.dk 


\section{Abstract}

G protein-coupled receptors (GPCRs) enable cells to sense environmental cues and are indispensable for coordinating vital processes including quorum sensing, proliferation, and sexual reproduction. GPCRs comprise the largest class of cell surface receptors in eukaryotes, and for more than three decades the pheromone-induced mating pathway in baker's yeast Saccharomyces cerevisiae has served as a model for studying heterologous GPCRs (hGPCRs). Here we report transcriptome profiles following mating pathway activation in native and hGPCR-signaling yeast, and use a model-guided approach to correlate gene expression to morphological changes. From this we demonstrate mating between haploid cells armed with hGPCRs and endogenous biosynthesis of their cognate ligands. Furthermore, we devise a ligand-free screening strategy for hGPCR compatibility with the yeast mating pathway and enable hGPCR-signaling in the probiotic yeast Saccharomyces boulardii. Combined, our findings enable new means to study mating, hGPCR-signaling, and cell-cell communication in a model eukaryote and yeast probiotics.

\section{Introduction}

From the first genetic studies of yeast (1), to the discovery of heterothallism (2) and identification of genes involved in mating (3), the baker's yeast Saccharomyces cerevisiae (S. cerevisiae) has been considered a dominant model organism for studying genetics (4) and, in recent decades, heterologous G protein-coupled receptor (hGPCR) signaling (5-8). The genome of $S$. cerevisiae encodes two GPCRs involved in mating, one for each of the two mating pheromones, namely STE2, which senses a-factor produced by MATa cells, and STE3, which senses a-factor produced by MATa cells (9). STE2 and STE3 are not essential and can be substituted with heterologous GPCRs (hGPCRs) for health and biotechnological applications (10), including large-scale drug discovery (11), point-of-care pathogen detection (12), directed 
evolution of hGPCRs (13), and as sensor-actuator probiotic agents in mouse models of disease (13). After successful hGPCR expression and G protein-coupling, cognate ligands can activate the yeast mating pathway, which constitutes an evolutionarily conserved mitogen-activated protein kinase (MAPK) pathway, recently minimized to simplify hGPCR deorphanization and signaling studies in $S$. cerevisiae (8). In brief, the $G_{a}$ subunits of the heterotrimeric $G$ protein physically bind to the native GPCRs, Ste2 and Ste3, serving as guanine-nucleotide exchange factors (GEFs). Upon ligand binding, Ste2 and Ste3 facilitate exchange of GDP for GTP on the $\mathrm{G}_{\alpha}$ subunit and subsequent $\mathrm{G}_{\beta y}$ release (10). This in turn activates the MAPK cascade orchestrating major transcriptome perturbations, cell cycle arrest, polarized growth, and ultimately mating $(9,14)$.

Despite the multitude of studies on hGPCR coupling to the yeast mating pathway $(8,10$, 11), there is lack of knowledge on both systemic signaling and mating in yeast upon substitution of native pheromone GPCRs for heterologous counterparts, constraining yeast as a model organism and limiting its application within biotechnology and health. So far, omics studies of mating pathway activation have focused on native pheromone-induced explorations (14-16), while investigations of the compatibility of hGPCRs with yeast mating have been limited to substituting only one of the yeast pheromone GPCRs at the time in MATa/MATa mating pairs (5, 17-19). Such substitutions of individual yeast GPCRs with heterologous yeast pheromone receptors have permitted mating, albeit at low frequencies $(\leq 1 \%)(18)$, while another seminal study reported that substitution of human $\beta_{2}$-adrenergic receptor $\left(\mathrm{h} \beta_{2}-\mathrm{AR}\right.$ ) in place of STE2 could not support mating (5). Even though largely unresolved, it is expected that establishing synthetic mating in a model eukaryote would deepen our understanding of how an evolutionary conserved MAPK signaling route, such as the yeast mating pathway, is regulated, as well as support studies of engineered cell-cell communication, mating behavior, and the development of high-throughput assays within biotechnology and medicine. 
Here we compare the transcriptome of strains engineered with different hGPCRs to wildtype and sensitized yeast devoid of the negative regulator of the mating pathway, SST2 (20), and in response to different dosages of their cognate ligands. We identify $>1,000$ differentially expressed genes and correlate RNA-seq data to biosensing of hGPCR ligands and mating morphology in S. cerevisiae, ultimately demonstrating that substituting native yeast GPCRs with signaling hGPCRs can enable efficient mating in yeast. Our study also describes a ligand-free assessment of functional hGPCR-coupling in yeast, and presents a simple approach to activate dormant GPCR-signaling in the probiotic yeast Saccharomyces boulardii.

\section{Results}

Biosensing yeast strain engineering and characterization

Before studying the impact of hGPCR expression at the systems level, we first characterized biosensing in $S$. cerevisiae of adenosine ( $A 2 \mathrm{bR})$, encoded by $A D O R A 2 B$, melatonin $\left(\mathrm{MT}_{1}\right)$, encoded by MTNR1A, serotonin (5-HT $\left.4 \mathrm{~b}\right)$, encoded by HTR4, and the fission yeast pheromone P-factor (Mam2), encoded by MAM2. The strain designs adhered to established literature $(6,8,11,21)$, in which genes encoding hGPCRs were overexpressed from the $C C W 12$ promoter, while the native $\mathrm{G}_{\alpha}$ subunit (GPA1), or a chimeric humanized $\mathrm{G}_{\alpha}$ subunit $\left(G P A 1 / G_{\alpha i 2}\right)$, were expressed from the weak (RNR2), medium (ALD6), or strong (PGK1) promoters in order to differentially tune mating pathway response to hGPCR-signaling. The strains further contained deletions of SST2, native pheromone receptors (STE2 and STE3), and an expression cassette for expression of green fluorescent protein (GFP) from the native pheromone-inducible FUS1 promoter (Fig. 1A, Suppl. Fig. S1A). In addition, the resulting biosensing strains retained coupling to the entire mating pathway, including FAR1 which is responsible for activation of G1 cell cycle arrest prior to mating (10). In accordance with previous studies $(8,11,21)$, we observed that the $P_{F U S_{1}^{-}}$GFP reporter was induced in all 
biosensing strains across a gradient of cognate ligand dosages (Fig. 1B), although we observed signaling from $5-\mathrm{HT}_{4 \mathrm{~b}}$ only in a narrow operational range for the $\mathrm{P}_{R N R 2}-G P A 1 / G_{\text {ai2 }}$ strain design (Suppl. Fig. S1B).

Intriguingly, in the absence of ligands, all four hGPCRs altered reporter expression up to 4-fold, solely from introduction to the $P_{R N R 2}$ GPA1 background strain (Fig. 1B). We termed this phenomenon a "coupling-shift" (see Discussion) and observed that coupling-shifts were also evident for 6 additional hGPCRs known to signal in yeast $(5,7,22,23)$, as well as for 3 hGPCRs not previously shown to signal in yeast (referred here as ZtSte2, TrSte2, and MsSte3) originating from pathogenic fungi, and for which cognate pheromones are currently not known (Suppl. Fig. S1C).

Thus, $P_{R N R 2}-G_{a}$ strain designs presented a simple pre-screen for putative coupling of hGPCRs to the yeast mating pathway, while the $P_{P G K 1^{-}}-G_{\alpha}$ strain designs allowed tight control of mating pathway activation.

\section{Transcriptome analysis of hGPCR-signaling yeast}

Activation of the pheromone-induced mating pathway in yeast evokes cell cycle arrest and cell differentiation, including expression changes in hundreds of genes (14). To elucidate transcriptome perturbations in yeast during hGPCR-signaling, and further guide efforts to successfully coupling of hGPCRs to the yeast mating pathway, we compared transcriptomes from biosensing strains expressing $\mathrm{A} 2 \mathrm{bR}, \mathrm{MT}_{1}$, or $5-\mathrm{HT}_{4 \mathrm{~b}}$, to wild-type and sensitized (sst2 $\Delta$ ) strains with and without cognate ligand stimulation. From this analysis, we identified 1,178 differentially expressed genes (DEGs) across all 20 experimental conditions (Suppl. Table S1). The wild-type and sensitized sst2 $\Delta$ strains solely account for $\sim 75 \%(878 / 1,178)$ of the DEGs, while $8 \%(92 / 1,178)$ of the DEGs were unique to strains expressing hGPCRs (Fig. 1C). Importantly, we identified a core set of 57 DEGs that overlapped between all strains and 
conditions (Fig. 1C \& 1D, Suppl. Table S1). Among this set of core DEGs, and in accordance with previous gene expression studies performed on wild-type yeast $(14,24)$, we identified numerous mating pathway genes, including MFA1/MFA2, FUS1/FUS2/FUS3, and FIG1, as well as over-represented gene ontologies related to receptor signaling and conjugation, in all five strains (Fig. 1D, Suppl. Fig. S1D). Expression of individual DEGs varied greatly between individual strains, with wild-type and sensitized sst2 $\Delta$ strains showing higher expression perturbations in response to their cognate ligand than was the case for strains expressing hGPCRs (Fig. 1D, Suppl. Table S1). For instance, neither FUS3, the kinase eliciting yeast mating pathway signal amplification, nor FUS1 or FUS2, which are involved in membrane fusion during mating (10), were induced to the same extent in any of the biosensing yeast strains as compared to wild-type or sensitized yeast strains (Fig. 1D, Suppl. Table S1). Vice-versa, only three DEGs were shared exclusively between the three strains expressing hGPCRs. These included induction of two genes controlling expression of proteins with putative functions (YFL065C and YCL121W-A) and STE6, required for a-factor export, while 10-53 DEGs were unique to strains expressing individual hGPCRs (Fig. 1C, Suppl. Table S1).

Interestingly, we noticed lower expression of chimeric $G P A 1 / G_{\text {ai2 }}$ as compared to GPA1 (Suppl. Table S2), although only the 5 C-terminal amino acids differed in these designs. Generally, while most hGPCR studies in yeast use constitutively active promoters to drive $G_{\alpha}$ expression in biosensing yeast $(6,8,25), G_{\alpha}$ expression should ideally be high in the absence of ligand, and subsequently decrease upon ligand recognition to facilitate $G_{\beta \gamma}$ release and enhanced signal transduction. We identified two such dynamically regulated promoter candidates, $P_{\text {YLI69C }}$ and $P_{\text {HPF1 }}$, among the 57 core DEGs for strains expressing $A 2 b R$ and $M T_{1}$ (Fig. 1D, Suppl. Table S1). Other DEGs relevant for future engineering of biosensing and actuation, such as $A G A 2, K A R 4$, and MFA1/MFA2, all showed $>10$-fold activation across all strains. Of these, MFA1/MFA2 displayed high basal activities in biosensing strains, while AGA2 and KAR4 showed basal activities lower than in the wild-type strain (Suppl. Table S1). 
In conclusion, our transcriptome analysis identified close to 900 DEGs shared only among the wild-type and sensitized sst2 $\triangle$ yeasts, a core set of 57 DEGs shared between wildtype, sensitized sst2 $\Delta$, and all biosensing yeasts, as well as several promoters dynamically regulated during hGPCR-signaling.

\section{A single genetic edit enables biosensing probiotic yeast}

Extending from our initial analyses, we next investigated if biosensing strain designs from $S$. cerevisiae could be transferred into probiotic yeast. For several decades, the yeast $S$. boulardii has been used to treat and prevent gastrointestinal disorders (26). However, hGPCRsignaling in $S$. boulardii has never been reported, even though this could have major implications to advance the engineering of yeast therapeutics (13). We hypothesized that the native pheromone GPCRs in $S$. boulardii do not signal because it exists in the diploid MATa/a state (27). Indeed, engineered MATa/a $S$. boulardii can produce viable offspring with $S$. cerevisiae (28), and it was realized decades ago that homozygous Saccharomyces MATa/a and MATa/a diploids are able to mate (29), altogether indicative of pheromone signaling in such diploids.

Thus, we first devised a sniper approach to make homozygous MATa/a diploid $S$. boulardii by targeting the MATa region for double-strand break with CRISPR/Cas9 (30), in a strategy mimicking native yeast mating-type switching (9) (Fig. 2A). The guide RNA that we used for this procedure targeted both MATa and HML, which contains a copy of the a genes required for the mating type switch. Therefore, activity of $S$. boulardii's $\mathrm{HO}$ endonuclease would not cause reversion to the MATa/a state. Following introduction of the $\mathrm{P}_{\text {FUS } 1}$-GFP reporter to these strains, we observed a 5-fold Ste2-mediated increased reporter output in presence of afactor, while the parental MATa/a strain did not respond to any concentrations of a-factor (Fig. 2B). 
To equip S. boulardii with the design principles for hGPCR-signaling that we learned from S. cerevisiae (Fig. 1A \& 1B, Suppl. Fig. S1A), we engineered S. boulardii MATa/a strains

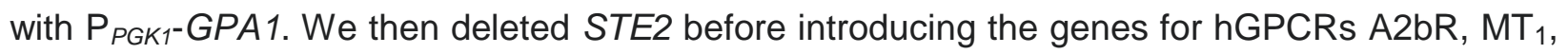
or Mam2, to test reporter output from $\mathrm{P}_{F U S 1}-$ GFP following incubation with increasing concentrations of cognate ligands. Here, we observed biosensing in all three probiotic strain designs (Fig. 2C-E). Specifically, A2bR-expressing MATa/a strains showed a modest, but significant, increase in reporter output from $10 \mu \mathrm{M}$ adenosine stimulation (1.3-fold) (Fig. 2C). Likewise, reporter output from $\mathrm{MT}_{1}$ - or Mam2-expressing strains increased after stimulation with melatonin (3-fold) or P-factor (4.5-fold), respectively (Fig. 2D \& 2E).

As in our biosensing $S$. cerevisiae strain designs (Fig. 1A, Suppl. Fig. S1A), we next deleted the negative mating pathway regulator, SST2, to increase reporter output (Suppl. Fig. S2A-C). However, this deletion reduced reporter outputs for all strains to modest maximum foldchanges of 1.5-2.0 fold upon stimulation with their cognate ligands (Suppl. Fig. S2A-C). As a further attempt to increase mating pathway activation, we replaced the strong $\mathrm{P}_{P G K 1}$ promoter driving expression of GPA1 with the weaker $P_{\text {RNR2. }}$. Here, we observed $>5$-fold increased reporter output from $100 \mu \mathrm{M}$ a-factor stimulation in MATa/a SST2 strains (Suppl. Fig. S2D). In contrast, in MATa/a sst2 $\mathrm{s}$ strains, $\mathrm{P}_{\text {RNR2 }}$ GPA1 completely nullified mating pathway signaling upon $\alpha$-factor stimulation, while $\mathrm{P}_{P G K 1}-G P A 1$ strain designs reduced background signaling and displayed 7- to 34-fold increased reporter output from a-factor stimulation (Suppl. Fig. S2D).

Taken together, pending simple mating-type switching, we have shown that biosensing strain designs from the model yeast $S$. cerevisiae are functional in probiotic $S$. boulardii, albeit necessitating further exploration of G protein balancing and hGPCR-signaling in this organism. 
Chemotropism and navigation along chemical gradients are universal traits of living organisms (31). In S. cerevisiae, shmooing is a hall-mark of chemotropism and inherent to mating pathway activation and successful cell-cell conjugation (3). In search of optimal parameters for hGPCR-mediated activation of the mating pathway, we initially tested if hGPCRsignaling yeast strains could shmoo across increasing concentrations of cognate ligands. Here, we defined shmoos from side-scatter (SSC-A) quantified by flow cytometry, which was validated using microscopy as previously described $(5,32)$. Multivariate analysis across all biosensing $S$. cerevisiae strain designs revealed a greater correlation between the $\mathrm{P}_{\text {FUS }}$-GFP reporter and SSC-A $\left(R^{2}=0.92\right)$ than with forward-scatter (FSC-A) $\left(R^{2}=0.81\right)$ (Fig. 3A, see Methods), previously used for shmoo quantification (33). Thus, the complex shmooing response, spanning $>4$ orders of magnitude of ligand concentrations, can be directly correlated to reporter fluorescence intensity.

We noticed that individual design parameters were important determinants of the observed correlation between shmooing and reporter fluorescence. Specifically, $P_{R N R 2}-G_{\alpha}$ designs displayed greater shmoo-fluorescence correlations compared to $P_{A L D 6}-G_{\alpha}$ and $P_{P G K 1}-G_{\alpha}$ designs $\left(R^{2}=0.97\right.$ vs $R^{2}=0.89$ and $R^{2}=0.88$, respectively) (Fig. 3B), although A2bR and $\mathrm{MT}_{1}$ designs exhibited strong correlations $\left(R^{2}=0.97\right)$ regardless of $G_{\alpha}$ expression design (Fig. 3C). Likewise, and in agreement with FUS1 being identified as a core DEG from our RNA-seq study (Fig. 1C \& 1D), the $P_{P G K 1}-G_{\alpha}$ strain designs with no or low background activation of the mating pathway (Fig. 1B) also showed strong correlations $\left(R^{2}=0.92-0.99\right)$ between shmooing and reporter fluorescence for all biosensing strains (Suppl. Fig. S3A). Microscopy confirmed the shmoo morphology interpreted from SSC-A (Fig. 3D \& 3E, Suppl. Fig. S3B). Lastly, increased SSC-A was also apparent for the $P_{P G K 1}-G_{\alpha}$ strains composed in MATa/a S. boulardii across gradients of cognate ligands (Suppl. Fig. S3C).

Altogether, and in agreement with classical reporter assays $(6,14)$, this data shows that mating pathway activation of hGPCR-expressing biosensing yeasts can be tightly controlled 
across large ligand gradients, depending on biosensing strain design, and correlates well with morphological changes indispensable for mating and cell-cell conjugation.

\section{Semi-synthetic mating in hGPCR-signaling yeast}

Having confirmed that hGPCR activation evokes shmooing in biosensing yeast strains, we next asked if this activation also translated into mating. In the following, we define mating between haploid MATa and MATa cells with single-receptor substitutions of STE2 in MATa cells as "semi-synthetic mating". As measures of sexual reproduction between engineered haploid cells we report both population-wise diploid frequencies and calculated mating efficiencies, with the former refering to the proportion of the entire sampled mating culture identified as diploids, while the latter describes how efficiently the given amount of diploids have formed relative to the amount of potential mating events (see Methods).

To investigate if biosensing strains expressing hGPCRs could support semi-synthetic mating, we initially substituted the wild-type MATa partner cell with a biosensing yeast strain for P-factor, serotonin, or melatonin to conduct mating trials with a MATa cell that retained its native Ste3 receptor. MATa cells were engineered to produce the synthetic pheromones (P-factor, serotonin, or melatonin) to mimic native paracrine signaling, while the biosensing yeast strains all retained native a-factor expression (Fig. 4A). In addition, we included a MATa mating partner without production to investigate if supplementation of cognate ligands would present a viable strategy to control mating as compared to endogenous production. Due to the low background activation of the mating pathway and morphological changes observed for strain designs with high expression of $G_{\alpha}$ (Fig. 1B, Fig. 3B \& 3D), we focused our attention on hGPCR-expressing strains with $P_{P G K 1}-G_{\alpha}$ designs.

We first evaluated the cognate ligand supplementation strategy without production for the adenosine biosensing strain. Here we observed a maximum mating efficiency of $29 \%$, which increased $~ 8$-fold from 0-100 $\mu \mathrm{M}$ adenosine supplementation (Fig. 4B). By comparison, wild- 
type MATa x MATa (positive control) and receptor-deficient MATa x MATa (negative control) crosses showed $40 \%$ and $3 \%$ mating efficiencies, respectively. Similarly, diploid formation from semi-synthetic mating based on supplementation of adenosine reached up to $12 \%$ of the screened population, whereas wild-type crosses amounted to $23 \%$ (Fig. 4B).

Having confirmed cognate ligand supplementation as a viable strategy for semi-synthetic mating, we next investigated mating efficiencies from endogenous production of synthetic pheromones with and without additional supplementation. Here, the biosensing strain expressing Mam2 crossed with a strain producing P-factor resulted in a mating efficiency of $22 \%$ without supplementation compared to $5 \%$ in crosses without production or supplementation (Fig. 4C). The same pattern was observed for crosses with strains producing melatonin or serotonin (Fig. 4D \& 4E, Suppl. Table S3), and the resulting diploids also displayed increased SSC-A compared to haploids, the extent of which was related to the hGPCR stimulation applied during mating (Suppl. Fig. S4A). Production of synthetic pheromones for crosses with P-factor and melatonin biosensing strains showed similar mating efficiencies compared with those observed for supplementation of cognate ligands (Fig. 4C \& 4D), whereas for the serotonin biosensing strains, high production outperformed the best mating efficiencies observed at $10 \mu \mathrm{M}$ supplementation alone (34\% vs 14\%) (Fig. 4E, Suppl. Table S3). Strikingly, supplementation of $100 \mu \mathrm{M}$ serotonin in the high-production crosses yielded reduced mating efficiency compared to lower concentrations of cognate ligand supplementation (Fig. 4E). This was also observed for P-factor supplementation in excess of $0.1 \mu \mathrm{M}$, irrespective of P-factor production (Fig. 4C), indicating a potential mating pathway overstimulation which could also be observed for these strains in the initial mating pathway characterization (Fig. 1B). Likewise, while this pattern was not observed for the dosages tested in this study for the A2bRand $\mathrm{MT}_{1}$-expressing strains (Fig. 1B, Fig. 4B \& 4D), the positive correlation observed between shmooing and mating efficiencies at ligand concentrations up to $10 \mu \mathrm{M}$ diluted at higher concentrations of supplemented ligands (Fig. 3E, Fig. 4C \& 4E), underscoring that even though 
shmooing is indispensable for mating and cell-cell conjugation, the size of shmoos are not the sole determinant of mating efficiency.

In sum, these results show that replacing Ste2 with human or fungal GPCRs in biosensing yeast strains enables efficient semi-synthetic mating, and that mating efficiencies do not directly relate to shmoo sizes, yet are controllable by cognate ligand supplementation and synthetic pheromone production.

\section{Full synthetic and autonomous yeast mating}

To further explore the contribution from each hGPCR in complete absence of native pheromone signaling, we substituted pheromone receptors in both mating partners for hGPCRs. As for semi-synthetic mating trials, we initially supplemented the cognate ligands exogenously, either individually or in combination for each mating pair (Fig. 5A).

Here, supplementation of adenosine and melatonin together revealed synergistic mating efficiencies starting at $1 \mu \mathrm{M}$ for each ligand. The highest mating efficiency was observed at 100 $\mu \mathrm{M}$ adenosine and melatonin combined (40\%), while $10 \mu \mathrm{M}$ of each ligand yielded the most frequent diploid formation (7.6\%) (Fig. 5B, Suppl. Fig. S5). When supplied individually, only melatonin clearly increased mating efficiencies, corroborating the results obtained in cells without melatonin production (Fig. 4D, Fig. 5B). Yet, the mating efficiencies and diploid formation frequencies observed from supplementation of individual ligands were never as high as for simultaneous stimulation with both cognate ligands.

In another mating cross with adenosine and serotonin biosensing strains, individually supplementing $10 \mu \mathrm{M}$ adenosine or serotonin resulted in elevated mating efficiencies only with serotonin supplementation (4.5\%-7.3\%) relative to no supplementation (3.6\%) (Fig. 5C). Supplementation of both ligands together at $10 \mu \mathrm{M}$ resulted in the highest mating efficiency (11\%) and diploid formation (4.5\%) for this mating pair (Fig. 5C). Conversely, in mating pairs expressing $\mathrm{MT}_{1}$ and $5-\mathrm{HT}_{4 \mathrm{~b}}$, we observed no increased mating efficiency from only 
supplementing serotonin, whereas melatonin supplementation alone again resulted in elevated mating efficiencies (3.6\%-6.6\%) (Fig. 5D). Importantly, and just as for the results obtained with supplementation of adenosine and melatonin in synthetic mating pairs expressing $A 2 b R$ or $\mathrm{MT}_{1}$

(Fig. 5B), supplementation of both ligands at $10 \mu \mathrm{M}$ gave the highest mating efficiency (10\%) (Fig. 5D). Furthermore, albeit with a different impact from each hGPCR in synthetic mating pairs, decreased diploid frequencies were apparent at the highest supplementation levels across all three biosensing yeast mating pairs (Fig. 5B-D). Finally, we investigated full autonomous mating between biosensing yeasts expressing Mam2 or $5-\mathrm{HT}_{4 \mathrm{~b}}$ with endogenous production of synthetic pheromones (P-factor and serotonin) (Fig. 5E). Here we observed the highest mating efficiency $(6.6 \%)$ in the mating pair producing both synthetic pheromones. Also, mating efficiency increased compared to the negative mating control when at least one of the synthetic pheromones was produced (Fig. 5E). Once again, resulting diploids showed altered SSC-A following hGPCR stimulation (Suppl. Fig. S4B \& S4C). Lastly, and worth noting, is that while the mating efficiency was lower for mating pairs in which only one synthetic pheromone was produced, compared to the efficiency observed for mating pairs producing both serotonin and P-factor, the diploid frequency was highest when only serotonin was produced (Fig. 5E).

Altogether, our results show that both pheromone receptors in a yeast mating pair can be functionally replaced for hGPCRs, and that the mating efficiency and diploid frequencies can be controlled by dosage of their cognate ligand pairs, and to a lower extent by only supplementing single synthetic pheromones. Most importantly, our data also demonstrate full paracrine signaling in a fully autonomous manner as demonstrated by mating between $5-\mathrm{HT}_{4 \mathrm{~b}^{-}}$ and Mam2-expressing haploid cells producing the synthetic pheromones P-factor and serotonin, respectively.

\section{Discussion}


This study covers systemic transcriptional perturbations upon hGPCR-signaling and mating pathway activation in yeast. It further demonstrates successful cell differentiation and autonomous synthetic sexual reproduction in a model eukaryote, as well as mating pathway activation in probiotic yeast, $S$. boulardii. It offers a new resource for exploring control of biosensing and cellular morphology by the use of regulatory elements in yeast expressing either native or heterologous GPCRs and $G_{a}$ proteins. Taken together, this study highlights crucial design parameters and environmental conditions to tune mating pathway activation, cellular differentiation, and synthetic mating.

With respect to recommended strain designs for mating pathway activation, we consider "coupling-shift" as a first feature to assess. By design, weak reporter expression is thought to arise from hGPCR-G ${ }_{\alpha}$ coupling, in which the hGPCR exerts GEF function on spontaneously arising nucleotide-free $G_{a}$ subunits that leads to ligand-independent signal transduction by pushing the equilibrium towards $G_{\beta y}$ release in sensitive biosensing yeast strains. Thus we envision future studies to leverage coupling-shifts for cheap ligand-free pre-screens to

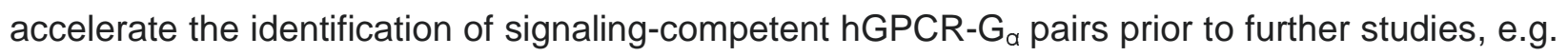
deorphanization. Likewise, tuning of $G_{\alpha}$ expression enables high-throughput assessment of nonlinear dosage-dependent shmoo sizes, thereby helping to determine optimal balancing of paracrine signaling for successful synthetic mating. Specifically, based on this study, we recommend expression of $G_{\alpha}$ using a weak promoter for assessing hGPCR coupling to the mating pathway, and a strong promoter for initial testing of synthetic mating as this design demonstrated low basal activity, high dynamic output ranges (up to 200-fold), as well as relatively large changes in shmoo sizes (Fig. 1B, Fig. 3E).

However, engineering of sexual reproduction at large needs careful considerations beyond choice of hGPCR, expression level of $G_{\alpha}$, and types of synthetic pheromones towards successful paracrine signaling, coordinated chemotropism, and mating. For instance, as evidenced from declining synthetic mating efficiencies and diploid formation from 
supplementation of synthetic pheromones at high concentrations (Fig. 4C \& 4E, Fig. 5C \& 5D), increased reporter expression during hGPCR-signaling does not necessarily capture mating pathway overstimulation (Fig. 1B, Fig. 3E). Also, even though shmoo sizes and mating efficiencies both declined at high dosages for the $P_{P G K^{1}}-G_{\alpha}$ design with $5-\mathrm{HT}_{4 \mathrm{~b}}$ (Fig. 4E), the same correlation was not apparent for the almost identical Mam2 strain design (Fig. 3E, Fig. 4C). Extending from these observations, establishment of optimal pheromone gradients for efficient cell-cell communication and mating using synthetic pheromones should greatly benefit from identification of new barrier proteins, or metabolic enzymes, analogous to pheromonebinding/-cleaving $A B F 1$ and BAR1 from MATa and MATa cells, respectively $(34,35)$.

Lastly, this study is to the best of our knowledge a first demonstration in the probiotic $S$. boulardii of mating pathway activation controlled by signaling from hGPCRs (Fig. 2), yet further studies are encouraged to elucidate the apparent different role that the negative regulator of mating pathway activation, SST2, plays in this conspecific yeast (Suppl. Fig. S2A-D) compared to $S$. cerevisiae. Indeed, from understanding the regulatory mechanisms of the mating pathway, and taking advantage of $S$. boulardii's growth, survival, and residence time in the gastrointestinal tract as compared to $S$. cerevisiae (36), we envision that probiotic $S$. boulardii can now be engineered to dynamically respond to disease markers for timely and accurate secretion of therapeutic compounds, and as such adds an important capability to its already established therapeutic potential. Extending from this we furthermore foresee this study to foster the development of growth-based high-throughput screens of hGPCR-mediated chemotropism within environmental engineering, MAPK pathway signaling, drug discovery for therapeutic purposes, and enzyme optimization for metabolic engineering and biotechnology.

\section{Acknowledgements}


This study is supported by grants from the Velux Foundations (28309) to EDJ and the Novo Nordisk Foundation (NNF20CC0035580 and NNF19CC0035454) to MKJ and BL, the National Science Foundation (CBET-1934284), seed funding from the National Institutes of Health (P30DK034987), and Startup Funds from North Carolina State University to NC, a T32 training grant to JMV (5T32GM008776), and a fellowship from the ministry of Higher Education Oman to ISA. We thank Dr. Nicholas Milne for providing plasmid pCfB9221. Schematic figure illustrations for panel $A$ in Figures 1 and 4, and for panels $A$ and $E$ in Figure 5, were created with BioRender.com.

\section{Competing interests}

The authors declare no financial interest

\section{Author contributions}

Emil D. Jensen: Project administration, equal, Conceptualization, lead, Writing - original draft, lead, Formal analysis, equal, Writing - review \& editing, equal, Funding acquisition, equal. Marcus Deichmann: Investigation, lead, Conceptualization, equal, Formal analysis, equal, Writing - review \& editing, equal. Xin Ma: Investigation, equal. Rikke U. Vilandt: Investigation, supporting. Giovanni Schiesaro: Investigation, supporting. Marie B. Rojek: Investigation, supporting. Bettina Lengger: Investigation, supporting. Justin M. Vento: Investigation, supporting. Deniz Durmusoglu: Investigation, supporting. Sandie P. Hovmand: Investigation, supporting. Ibrahim Al'Abri: Investigation, supporting. Jie Zhang: Investigation, supporting. Nathan Crook: Conceptualization, supporting, Funding acquisition, supporting. Writing - review \& editing, supporting. Michael K. Jensen: Project administration, equal, Conceptualization, equal, Writing - original draft, equal, Writing - review \& editing, equal, Funding acquisition, equal. All authors approved the manuscript. 


\section{Materials and Methods}

\section{Molecular cloning}

Standard techniques and materials: USER cloning (37) was used to construct plasmids, unless otherwise specified, and the EasyClone MarkerFree system with integration plasmids and gRNA plasmids compatible with CRISPR/Cas9 (38) were used throughout. USERcompatible vectors were treated with FastDigest SfaAl (ThermoFisher Scientific) and Nb.Bsml (NEB) prior to ligation, and USER-compatible fragments were amplified with Phusion U Hot Start PCR Master Mix (ThermoFisher Scientific) to read through uracil overhangs contained in oligos. Strains, plasmids, oligos, gene blocks, and heterologous GPCR accession IDs are listed in Suppl. Tables S4-S8.

Codon optimization of gene blocks: Gene blocks gDAM5 and gDAM8-14 were codon optimized for Saccharomyces cerevisiae with the IDT Codon Optimization tool. All oligos and gene blocks were purchased from IDT, and coding sequences were immediately preceded by a transcriptional enhancer sequence (AAAACA).

gRNA plasmids: Single gRNAs were introduced to existing gRNA plasmids by inverse PCR and blunt-end ligation with T4 DNA ligase (NEB). Oligos DAM1-4 and DAM6 were used individually with DAM594 to amplify pCfB3050 making up plasmids pDAM1-4 and pDAM6. pDAM7 and pDAM8 were made by amplifying the gRNA cassettes from pDAM1 and pDAM2 with, oligos TJOS-62 and TJOS-65 and assembled into pEDJ400 and pEDJ437 (39), respectively. Oligos DAM218+DAM594 and DAM219+DAM594 amplified pDAM1 to give plasmids pDAM77 and pDAM78, DAM485+DAM594 amplified pDAM8 to make pDAM182, and DAM335+DAM594 amplified pDAM7 to make pDAM146. gRNA cassettes from pDAM77 and pDAM78 were amplified with TJOS-62+TJOS-66 and TJOS-63+TJOS-65 (40), respectively, and assembled into pEDJ437 to give pDAM82. Plasmids ID6911 and PL_12_I3 were made from 
inverse amplification of pDAM7 with oligos JZ1+DAM594 and MAD1+DAM594, respectively. pDAM135 was created by amplification of the PL_12_I3 cassette using TJOS-62+TJOS-65 and assembly into pEDJ437. TJOS-62+TJOS-66, TJOS-63+TJOS-67, TJOS-64+TJOS-65 amplified pCfB3042, pCfB3045, and pCfB3049 gRNAs, respectively, and were assembled together into pTAJAK-71 (40) to make the triple gRNA plasmid pDAM22. pDD110 was made with Bsal golden gate cloning by assembly of the gene block DDgb008 and YTK003 (Con2), YTK010 (pCCW12),YTK036 (Cas9), DDgb008 (pSNR52, GFP dropout, sgRNA, tSUP4), YTK055 (tENO2), YTK071 (Con5), YTK078 (NatR), YTK081 (CEN6/ARS4), YTK084 (KanR-ColE1) according to protocol for the YTK toolkit (41). gRNA sequences were ordered as annealed oligos (DDpr262+DDpr263 for gRNA_URA3 and DDpr264+DDpr265 for gRNA_HIS3) with 4 bp overhangs, and with golden gate cloning entered into pDD110 to make pDD111 and pDD115.

$G_{\alpha}$ plasmids construction: Promoters $P_{R N R 2}, P_{A L D G}$, and $P_{P G K 1}$ were amplified from yeast genomic DNA (gDNA) with oligos EDJ134+DAM116, DAM117+DAM118, and DAM58+EDJ318, respectively. GPA1 was amplified with oligos DAM81+DAM82 from CEN.PK2-1C gDNA, and GPA1/ $\mathrm{G}_{\text {ai2 }}$ from gene block gDAM1 with oligos DAM81+DAM83. $\mathrm{P}_{P G K 1}, \mathrm{P}_{\text {RNR2}}$, or $\mathrm{P}_{A L D 6}$ were then assembled with GPA1 or GPA1/G ai2 into pRS415U to give plasmids pDAM30, pDAM32, pDAM47, pDAM49, pDAM50 and pDAM52 respectively.

hGPCR plasmid constructions: The promoter $\mathrm{P}_{\text {CCW12 }}$ was amplified from gDNA with

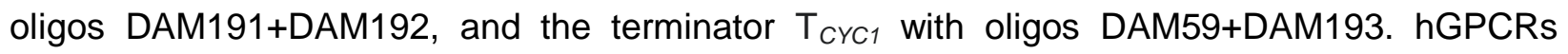
A2bR (ADORA2B), $\mathrm{MT}_{1}(M T N R 1 A)$, Mam2 (8), and 5-HT $4 \mathrm{~b}$ (HTR4) (21) were amplified from gene blocks gDAM2-5 with oligos DAM63+DAM64, DAM65+DAM66, DAM67+DAM68, and DAM160+DAM162, respectively, and each fragment was USER assembled with $\mathrm{P}_{\text {CCW12 }}$ and $\mathrm{T}_{C Y C 1}$ into pEDJ437 to give plasmids pDAM71, pDAM72, pDAM74, and pDAM76, respectively. Plasmids pDAM216-223 expressing hGPCRs h $\beta_{2}-A R$ (ADRB2) (5), CXCR4 (42), GLP-1R (23), CaSte2, FgSte2 or ZtSte2 (7), and TrSte2 (Uniprot ID: A0A022VRI2) or MsSte3 (Uniprot ID: 
A0A1M8A1X3), individually encoded in gDAM7-14, were amplified with oligos DAM335DAM350, respectively, and assembled as before with $\mathrm{P}_{C C W 12}$ and $\mathrm{T}_{C Y C 1}$ into pEDJ437.

Plasmids for synthetic pheromone production - serotonin, melatonin, and P-factor: All expression cassette plasmids used as templates for PCR have been previously described (43). pDAM23 ( $\left.\mathrm{T}_{A D H 1}-R_{n P T S}<-\mathrm{P}_{\mathrm{TEF1} 1^{-}} \mathrm{P}_{P G K 1^{-}}-\mathrm{RnSPR}-\mathrm{T}_{C Y C 1}\right)$ was made from amplification of pCfB1251

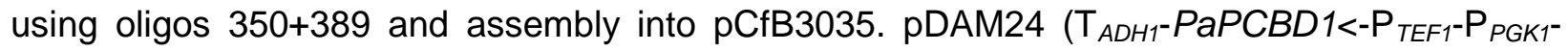
$>$ RnDHPR-T CYC1 ) was made from amplification of pCfB1248 using oligos $2149+2153$ and assembly into pCfB3040. To generate pDAM25 ( $\left.\mathrm{T}_{A D H 1^{-}}-H s A S M T_{<-\mathrm{P}_{T E F 1^{-}}-\mathrm{P}_{P G K 1^{-}}>\text {BtAANAT- }} \mathrm{T}_{C Y C 1}\right)$, BtAANAT was amplified from pCfB2628 using oligos 1761+1762, HsASMT from pCfB1252 using oligos $2254+2255$, as well as bidirectional joint promoters $\mathrm{P}_{T E F 1}-\mathrm{P}_{P G K 1}$ from pCfB2628 using oligos 5+8, and subsequently all parts were assembled into pCfB2899. pCfB9221 contains $\mathrm{T}_{A D H 1^{-}}-H s D D C<-\mathrm{P}_{T D H 3^{-}}-\mathrm{P}_{T E F 1^{-}}>S m T P H-\mathrm{T}_{C Y C 1}$.

Other plasmids: The Cas9 expression cassette from pEDJ391 was amplified with oligos 1564+EDJ325 and USER assembled into pRS416U to make plasmid pDAM215. mKate2 was amplified from pYR11 (44) with oligos DAM288+DAM289 and assembled with P $_{T E F 1}$ amplified from gDNA with oligos $1564+1565$ to make plasmid pDAM122. pDAM123 was made by assembling fragments from gDAM15 (a-leader secretion signal and P-factor) amplified with DAM76+DAM78, $P_{T E F 1}$ amplified with 1564+1565, and $T_{C Y C 1}$ amplified with DAM80+DAM81, into pCfB2899. Plasmid pJV452 was constructed by Gibson (NEB) assembly of pEDJ400 linearized with oligos JV498+JV499, which excluded the $2 \mu$ element, and CEN/ARS from plasmid DD118 (36) amplified with oligos JV500+JV501. Plasmid pDAM194 containing $\mathrm{P}_{F U S 1}-y E G F P-\mathrm{T}_{F U S 1}$ was made by amplification of gDNA from strain CPK16 with oligos JV502+JV503, which was then assembled into plasmid pJV452.

\section{Engineering of S. cerevisiae}


Strain CEN.PK2-1C and BY4741 (EUROSCARF) were transformed with pEDJ391 (39) to express Cas9 (CPK1 and SBY1). 1-2 $\mu$ g for each plasmid or fragment DNA was used in all chemical transformations of $S$. cerevisiae.

Baseline S. cerevisiae strains: CPK1 and SBY1 were consecutively genetically deleted for SST2, STE2, and STE3 with gRNA plasmids pDAM4, pDAM7, and pDAM8 and homology templates amplified as two overlapping fragments from gDNA with oligos DAM19-22 for sst2A, DAM7-10 for ste2A, and DAM13-16 for ste3A to give strains CPK2-4 and SBY2-4, respectively. In addition, a landing-pad-based strain was engineered using the same approach but with amplification of landing-pads from yWS677 (8) with oligos DAM19+DAM22, DAM8+DAM10, and DAM13+DAM16 for sst2 $\Delta$; ste2 $\Delta$; ste3 $\Delta$, respectively, resulting in platform strain CPK88. Next, the pheromone-responsive FUS1 promoter and terminator were amplified with oligos DAM5356. Both fragments included overlapping homology to yeast-enhanced green fluorescent protein ( $y E G F P)$ amplified from the gene block gDAM6 with oligos DAM39+DAM40. Co-transformation of these three fragments and the gRNA plasmid pDAM3 into strain CPK4 and SBY4 replaced the native FUS1 open reading frame with $y E G F P$ to give CPK16 and SBY16, respectively.

$\mathrm{G}_{\alpha}$ engineering and balancing of baseline $S$. cerevisiae strains: pDAM47 and pDAM49 were amplified with oligos DAM209+DAM212 to give the fragments $P_{R N R 2}-G P A 1-T_{C Y C 1}$ and $\mathrm{P}_{\text {RNR2- }}$ GPA1/ $\mathrm{G}_{\text {ai2- }} \mathrm{T}_{C Y C 1}$, pDAM50 and pDAM52 were amplified with oligos DAM210+DAM212 to give the fragments $P_{A L D \sigma}$ GPA1- $T_{C Y C 1}$ and $P_{A L D G}$ GPA1/G $G_{\alpha i 2}-T_{C Y C 1}$, and pDAM30 and pDAM32 were amplified with oligos DAM211+DAM212 to give the fragments $P_{P G K 1}-G P A 1-T_{C Y C 1}$ and $P_{P G K 1}-G P A 1 / G_{a i 2^{-}} T_{C Y C 1}$, respectively. CPK16 was transformed with each of these fragments individually and with the gRNA plasmid ID6911 for genome integration to make strains CPK125130. In the same way, SBY16 was engineered with integration of $P_{R N R 2}-G P A 1-T_{C Y C 1}$ to make strain SBY108, CPK88 was transformed with $\mathrm{P}_{P G K 1}-G P A 1-\mathrm{T}_{C Y C 1}$ and $\mathrm{P}_{P G K 1}-G P A 1 / G_{a i 2^{2}}-\mathrm{T}_{C Y C 1}$ for CPK88, resulting in strains CPK109 and CPK112, respectively, and SBY4 with $\mathrm{P}_{P G K 1^{-}}$GPA1$\mathrm{T}_{\text {CYC1 }}$ leading to strain SBY104. CPK125-130 and CPK109, CPK112, SBY104, and SBY108 
were genetically deleted for native GPA1 by co-transformation of gRNA plasmid pDAM82 and gDNA amplicons made with oligos DAM85+DAM235 and DAM34+DAM236, yielding strains CPK131-136, CPK115, CPK118, SBY116, and SBY120, respectively. CPK1, CPK115 and CPK118 were transformed with the super-folding GFP $\left(\mathrm{P}_{T E F 1}-S f G F P-T_{C Y C 1}\right)$ integration cassette purified from Notl digested pEDJ26 (45), and the gRNA plasmid pCfB3050, resulting in strains CPK46, CPK121 and CPK124. SBY116 was transformed with $\mathrm{P}_{\text {TEF1 }_{1}-\text { KKate2-T }_{C Y C 1} \text { from Notl }}$ digested pDAM122, and the gRNA plasmid pCfB3048, resulting in strain SBY128. To generate SBY53, XII-2 upstream homology, $\mathrm{P}_{T E F 1}, m R u b y 2, \mathrm{~T}_{A D H 1}$, and XII-2 downstream homology were amplified with DAM39-48, respectively, from gDNA and gDAM16, and co-transformed with pCfB3048 in SBY1 for genome integration. To edit the five last amino acids in Gpa1, CPK131 or SBY120 were transformed with the gRNA plasmid pDAM6, targeting the GPA1 C-terminal, and two overlapping homology fragments together comprising the new $G P A 1 / G_{a}$ sequence. Homology fragments were amplified from pDAM30 with the oligo combinations listed below to make resulting strains show in parentheses:

GPA1/G ${ }_{\alpha(L C G L I):} \mathrm{DAM} 31+\mathrm{DAM} 419$ and DAM418+DAM212 (CPK343), GPA1/G ${ }_{\alpha(D S G I L):}$ DAM31+DAM423 and DAM422+DAM212 (CPK347), GPA1/G ${ }_{\alpha(E T G F L):} \mathrm{DAM} 31+\mathrm{DAM} 427$ and DAM426+DAM212 (CPK350), GPA1/G ${ }_{\alpha(M C G L I):} \mathrm{DAM} 31+\mathrm{DAM} 551$ and DAM550+DAM212 (CPK424), GPA1/G ${ }_{a s(Q Y E L L):}$ DAM31+DAM37 and DAM38+DAM212 (SBY123).

DAM35+ID904 amplified fragments at $~ 900$ bp from the genomes of the resulting strains that contain $G P A 1 / G_{\alpha}$, which were sent for Sanger sequencing with DAM31 for sequence verification.

Mating-type switch in S. cerevisiae with CRISPR/Cas9: SBY53 and SBY128 were mating-type switched from MATa to MATa with gRNA plasmid pDAM135 to give SBY55 and 
SBY172. Mating-type switch was confirmed by PCR on ultrapure gDNA (Yeast DNA Extraction Kit 78870, ThermoFisher Scientific) with oligos PR_26_D7+PR_26_D8. Band sizes of $1.0 \mathrm{~kb}$ and $1.2 \mathrm{~kb}$ indicated MATa and MATa, respectively.

Genome integration of heterologous GPCRs in baseline S. cerevisiae strains: hGPCR expression cassettes were gel purified from respective plasmids following Notl digest and prior to transformation with gRNA plasmid pCfB3044. The purified pDAM71, pDAM72, and pDAM74 cassettes were each integrated in CPK131-133 to give CPK153-161, respectively, and in CPK121 to give CPK139, CPK142 and CPK331, respectively. The purified pDAM76 cassette was integrated in CPK124 and CPK134-136 to give CPK152 and CPK165-167, respectively. Purified cassettes from pDAM219-223 were each integrated in CPK131 to yield CPK450-454, respectively. The pDAM217 cassette was integrated in CPK134 to make CPK455, and purified cassettes from pDAM221 and pDAM223 were additionally integrated in CPK424 and CPK350, respectively, to make CPK456 and CPK459, respectively. The pDAM222 cassette was additionally integrated in CPK343 and CPK347 to make CPK457 and CPK458, respectively. Purified cassettes from pDAM218 and pDAM216 were each integrated in SBY123 and resulted in SBY143 and SBY146, respectively. Purified cassettes from pDAM72, pDAM71, and pDAM74 were integrated in SBY172 to give SBY156, SBY157, and SBY173, respectively.

Construction of $S$. cerevisiae ligand production strains - serotonin, melatonin, and Pfactor: Melatonin and serotonin producing strains were generated by genome integration of biosynthetic pathways essentially as previously described (43). Specifically, serotonin producing strains were created by one-pot integration of Notl-digested pDAM23, pDAM24, and pCfB9221, using gRNA plasmid pDAM22. The integrations were done in SBY55 and SBY173 to give SBY91 and SBY174, respectively. The melatonin biosynthetic pathway extends directly from the serotonin pathway. Melatonin producing strain SBY92 was created from SBY91 by additional

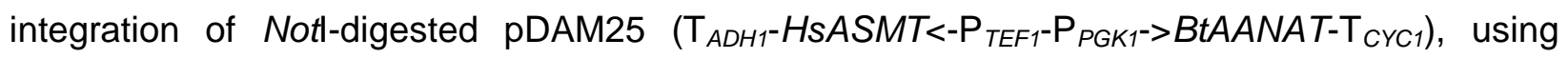
gRNA plasmid pCfB3020. Additionally, both serotonin and melatonin production was enhanced 
by multicopy random integration of Ty2-LoxP-KIURA3-TAG-P $P_{\text {GK } 1}-S m T P H-T_{C Y C 1}$ in Ty2 retrotransposon sites by transformation of Notl-digested pCfB2772. Screening of colonies by supernatant biosensing assays was conducted to identify highest producers, as described for "Supernatant Assays - Quantification of Synthetic Pheromones Production Levels". This engineering was done in strain SBY91, SBY92, and SBY174 resulting in SBY138, SBY139, and SBY175, respectively. P-factor producing strains were created by integration of Notl-digested pDAM123 using gRNA plasmid pCfB3020, which was done in strain CPK152 and SBY55, resulting in CPK448 and SBY155, respectively.

Strain engineering of $S$. boulardii: Construction of ura3/his3 auxotrophies: Transformation, Incubations, and recovery after transformation were done at $37{ }^{\circ} \mathrm{C}$ as previously described (36). S. boulardii ATCC MYA-796 (Sb.MYA-796) was co-transformed with $1 \mu \mathrm{g}$ of gRNA_URA3+Cas9 plasmid pDD111 and $1.5 \mu \mathrm{g}$ of repair template DDgb009 amplified with oligos DDpr273 and DDpr274. Transformants recovered in YPD and were plated on YPD+Nat $(100 \mu \mathrm{g} / \mathrm{ml})$. Resulting colonies were screened with colony PCR using oligos DDpr254+DDpr255. The band size for successful URA3 deletion was 430 bp and resulted in strain DD277. DD277 was then co-transformed with $1 \mu \mathrm{g}$ of gRNA_HIS3+Cas9 plasmid pDD115 and $1.5 \mu \mathrm{g}$ of repair template DDgb011 amplified with oligos DDpr276 and DDpr277. Transformants were recovered in YPD and plated on YPD+Nat $(100 \mu \mathrm{g} / \mathrm{ml})$. After 4 days, colonies were screened with colony PCR using oligos DDpr097+DDpr098. The band size for successful HIS3 deletion was $680 \mathrm{bp}$. Resulting strain DD313 was additionally verified for no growth on SC-UH dropout plates.

Genetic deletions and $G_{a}$-balancing of $S$. boulardii: All genetic modifications were performed exactly as described for S. cerevisiae in sections "Baseline S. cerevisiae strains" and " $G_{\alpha}$-balancing and engineering of baseline S. cerevisiae strains". Strain DD313 was transformed with plasmid pDAM215 to express Cas9 (SB4) and was then genetically deleted for SST2 (SB5) and STE3 (SB6). SB6 and SB4 were engineered to express $P_{P G K 1}-G P A 1-T_{C Y C 1}$ (SB19 and 
SB21, respectively) or $\mathrm{P}_{\text {RNR2 }}$-GPA1-T $\mathrm{T}_{C Y 1}$ (SB20 and SB22, respectively), which were all genetically devoid of native GPA1. STE2 was genetically deleted in strains SB19 and SB21, which resulted in SB23 and SB25, respectively.

Homozygous mating-type switch in $S$. boulardii with CRISPR/Cas9: Homozygous MATa/a mating-type switches were verified with oligos PR_26_D7+PR_26_D8 as described for S. cerevisiae above. SB4, SB5, SB19-22, SB23, and SB25 were each transformed with $1 \mu \mathrm{g}$ of gRNA plasmid pDAM182 to make strains SB8, SB9, SB30, SB24, SB35, SB26, SB31, and SB33, respectively.

Reporter and hGPCR expression plasmids transformations: $1 \mu \mathrm{g}$ of plasmid pDAM194 with the pheromone-responsive $\mathrm{P}_{F U S 1}-y E G F P-T_{C Y C 1}$ reporter construct was transformed in strains DD313, SB8, SB9, SB30, SB24, SB35, SB26, SB31, and SB33 to make strains SB14, SB17, and SB36-42, respectively. Next, $2 \mu \mathrm{g}$ of hGPCR expression plasmids pDAM71, pDAM72, or pDAM74 were transformed in SB41 to make SB45-47, respectively, and in SB42 to make SB48-50, respectively.

\section{Experimental procedures}

Handling of synthetic pheromones and ligands: All pheromone and ligand solutions used for dose-response and transcriptome analyses were made as $10 \mathrm{X}$ concentrated stocks and never exceeded 1\% DMSO content in culture (1X). Yeast survival and growth is not significantly affected at $<1 \%$ DMSO (46). Adenosine ( $\geq 99 \%$, Sigma-Aldrich), melatonin ( $\geq 98 \%$, SigmaAldrich), and serotonin hydrochloride ( $\geq 98 \%$, Sigma-Aldrich) were dissolved in DMSO (>99.9\%, Sigma-Aldrich) to a concentration of $100 \mathrm{mM}$, then 10 -fold diluted in SC media to $10,000 \mu \mathrm{M}$ $(10 \% \quad$ DMSO). P-factor (TYADFLRAYQSWNTFVNPDRPNL) and a-factor 
(WHWLQLKPGQPMY) (Custom Peptide Synthesis, 4 mg, $\geq 95 \%$ purity, GenScript Biotech)

were dissolved in DMSO to a concentration of $10 \mathrm{mM}$, then 10-fold diluted in SC media to 1,000

$\mu \mathrm{M}(10 \% \mathrm{DMSO})$. Then a 10 -fold dilution series using SC media $+10 \%$ DMSO was done to obtain a concentration range of $0.1-1,000 \mu \mathrm{M}$ for all pheromones and ligands. All solutions were stored at $-20{ }^{\circ} \mathrm{C}$. For mating trials, serotonin hydrochloride, melatonin, adenosine, and combinations hereof, were dissolved in SC medium in concentrations of $1,000 \mu \mathrm{M}$ without DMSO. A 10-fold dilution series was then made to obtain a concentration range of 1-1,000 $\mu \mathrm{M}$.

P-factor was dissolved in DMSO and diluted in SC media, whereafter a 10-fold dilution series was done in SC media $+1 \%$ DMSO to provide a concentration range of $0.1-10 \mu \mathrm{M}$ P-factor.

Bright-field microscopy: Bright-field microscopy was conducted on a Leica DM4000 B microscope (Leica Microsystems) equipped with a DFC300 FX camera (Leica Microsystems).

Dose-response and morphological analyses in $S$. cerevisiae and $S$. boulardii: Characterization of hGPCR-signaling was done by conducting dose-response analyses. $S$. cerevisiae and S. boulardii strains, incubated at $30^{\circ} \mathrm{C}$ and $37^{\circ} \mathrm{C}$, respectively, were inoculated in $0.5 \mathrm{ml} \mathrm{SC}$ or SC-UH media, respectively, for initial growth (24 hrs, $250 \mathrm{RPM}$ ). Overnight strains were diluted 10 -fold by addition of $4.5 \mathrm{ml}$ fresh media for further growth (20 hrs, 250 RPM). Prior to ligand stimulation, all cultures were adjusted to $\mathrm{OD}_{600}=0.2$ in fresh media, using a P300 NanoPhotometer® (Implen), and allowed brief growth (2 hrs, 250 RPM). To set up ligand stimulation in 96 deep-well plates, $180 \mu$ l culture was mixed with $20 \mu \mathrm{l}$ of each of the $10 \mathrm{X}$ ligand stocks resulting in final concentrations of $0-100 \mu \mathrm{M}$ of the cognate ligands in $1 \%$ DMSO. Cultures when then incubated for sensing of ligand (4 hrs, 250 RPM). Signal output from $\mathrm{P}_{F U S 1^{-}}$ 
GFP was quantified with flow cytometry. $30 \mu \mathrm{l}$ of each culture was diluted in $120 \mu \mathrm{l} 1 \mathrm{X}$ phosphate-buffered saline (PBS, Life Technologies) before sampling. S. cerevisiae strains were sampled on a BD LSRFortessa ${ }^{\mathrm{TM}} \mathrm{X}-20$ (BD Biosciences) flow cytometer, and S. boulardii strains on a NovoCyte Quanteon ${ }^{\mathrm{TM}}$ (Agilent) flow cytometer. For each condition, three biological replicates were analyzed, with a threshold of 10,000 events per replicate.

Supernatant Assays - Quantification of Synthetic Pheromones Production Levels: Production strains were inoculated in $0.5 \mathrm{ml} \mathrm{SC}$ media for initial growth $\left(24 \mathrm{hrs}, 30{ }^{\circ} \mathrm{C}, 250\right.$ RPM). Production cultures were then set up by replacing culture media by pelleting (2000 RPM, $3 \mathrm{~min}$ ) and resuspension in $500 \mu \mathrm{l}$ fresh SC. Cultures were then incubated for production in 96 deep-well plates, which for HPLC was done with an initial $\mathrm{OD}_{600}=0.2\left(24 \mathrm{hrs}, 30{ }^{\circ} \mathrm{C}, 250 \mathrm{RPM}\right)$ and for biosensing an initial $\mathrm{OD}_{600}=2.0\left(5 \mathrm{hrs}, 30^{\circ} \mathrm{C}, 250 \mathrm{RPM}\right)$. To acquire the pure media supernatant for quantification of ligand production, cells were removed by pelletting (4000 RPM, 5 min) and extraction of supernatant by pipetting, twice. HPLC analysis of serotonin and melatonin amounts produced by yeast strains was done on the Thermo Scientific ${ }^{\mathrm{TM}}$ UltiMate $^{\mathrm{TM}}$ 3000 HPLC using the Agilent Zorbax C18 4.6×100mm 30l5-Micron column with a Phenomenex AFO-8497 filter. Solvent A was $0.05 \%$ acetic acid, solvent B Acetonitrile. The solvent gradient applied was as follows:

\begin{tabular}{|l|l|l|l|}
\hline Time & Flow $[\mathrm{ml} / \mathrm{min}]$ & $\% \mathrm{~A}$ & $\% \mathrm{~B}$ \\
\hline 0 & 1.0 & 95 & 5 \\
\hline 1.5 & 1.0 & 88 & 12 \\
\hline 2.5 & 1.0 & 88 & 12 \\
\hline 4.5 & 1.0 & 70 & 30 \\
\hline 5.5 & 1.0 & 70 & 30 \\
\hline 8.0 & 1.0 & 30 & 70 \\
\hline 9.0 & 1.0 & 30 & 70 \\
\hline
\end{tabular}




\begin{tabular}{|l|l|l|l|}
9.5 & 1.0 & 95 & 5 \\
\hline 11.0 & End & End & end \\
\hline
\end{tabular}

Data analysis was done using Chromeleon ${ }^{\mathrm{TM}}$ Chromatography Data System (CDS) Software. Serotonin and melatonin values of samples were determined according to standard curves of serotonin hydrochloride and melatonin, in the range of 10 to $150 \mathrm{uM}$ and 2 to $20 \mathrm{uM}$ respectively. Serotonin peaks were detected at $1.920 \mathrm{~min}$, while melatonin peaks were seen at $7.037 \mathrm{~min}$.

\section{Mating trials}

Mating trials were based on fluorescent detection of mated and haploid cells by flow cytometry. This was accomplished by always partnering SBY-strains expressing mRuby2 or mKate2 with CPK-strains expressing sfGFP, providing a double-fluorescent signal from diploid cells. Firstly, strains were individually inoculated in $0.5 \mathrm{ml} \mathrm{SC}$ for initial growth $\left(24 \mathrm{hrs}, 30{ }^{\circ} \mathrm{C}\right.$, 250 RPM), then strains were diluted 10 -fold for further growth ( $22 \mathrm{hrs}, 30^{\circ} \mathrm{C}, 250 \mathrm{RPM}$ ). To remove accumulated pheromones of the pre-mating cultures, media was removed by pelletting (4000 RPM, 4 min.) and resuspended in fresh SC media without DMSO. To set up the mating trials, the cell densities of individual cultures were first determined by flow cytometry: $10 \mu \mathrm{l}$ culture was diluted in $190 \mu \mathrm{l}$ 1X PBS and run on a NovoCyte Quanteon and volumes containing $1,000,000$ cells were then calculated. The individual strains were then mixed to form co-cultures at an initial $1: 1$ ratio of $1,000,000$ cells of each of the two mating strains into a volume of $200 \mu l$ SC media per replicate (i.e. total of 10,000 cells/ $/$ l) in 96 deep-well plates. At this step, media containing synthetic pheromone or ligand was supplemented during resuspension. All mating trials had $5 \mathrm{hrs}$ incubation time at $30^{\circ} \mathrm{C}$ and 250 RPM. After mating, the co-cultures were examined by flow cytometry with $30 \mu \mathrm{l}$ culture diluted in $120 \mu \mathrm{l} 1 \mathrm{X}$ PBS sampled on a NovoCyte Quanteon $^{\mathrm{TM}}$ (Agilent) flow cytometer, and diploid selective plating was done on SC-UW. All 
mating trials were done in five replicates, with a threshold of 50,000 events per replicate.

\section{Data analysis and statistical analysis}

All analyses are detailed in Suppl. Tables S9-14.

Flow cytometry data and gating: All flow cytometry data was extracted as FCS files, and gated in FlowLogic ${ }^{\mathrm{TM}}$ v8.3 (Inivai Technologies). SSC-A, FSC-A, and fluorescence data points were derived from median values and median fluorescence intensities (MFI) of gated populations. Normalized MFI (nMFI) was obtained by normalizing to the mean of background MFIs. For statistics and data analysis means of medians and MFIs were applied \pm standard deviation. Population proportions were derived directly from event counts per gate, including diploid frequencies in mating trials. For $\mathrm{P}_{F U S 1}$-GFP-based hGPCR characterization and biosensor assays, small non-responsive cells were removed by consistent exclusive gates based on a minimum FSC-A values. In the case of normal-sized, but non-responsive, cells, their distinct autofluorescence was used to create an exclusive gate (B-530/30 vs Y-615/20). For mating trials, firstly, compensation was done between $\mathrm{Y}-615 / 20$ and B-530/30 using CompLogic automatic compensation in FlowLogic ${ }^{\mathrm{TM}}$ v8.3 (Inivai Technologies). Secondly, singlets were gated based on SSC (SSC-A vs SSC-H). Lastly, a quadrant gate was applied in the doublefluorescence dimension ( $\mathrm{Y}-615 / 20$ vs $\mathrm{B}-530 / 30)$ to classify all detected cells as either identifiable haploid $A$, haploid $B$, or diploid, or alternatively unidentifiable unknown cells lying below fluorescence thresholds. The quadrant gate was established by employing fluorescence minus one (FMO) controls.

Mating efficiencies and diploid frequencies: Diploid frequencies are the proportion of the entire sampled mating culture that are identified as diploids. Mating efficiencies describe how efficiently the given amount of diploids have formed relative to the amount of potential mating events. In this study mating efficiency is determined by the limiting haploid's method, which assumes that each diploid must have originated from exactly one cell of each of the two mated 
haploids. With this assumption, the amount of diploids that can be formed is constrained by the haploid with fewest available cells for mating - i.e. the limiting haploid. Applied to flow cytometric mating assays using double-fluorescence, this translates to one singlet double-fluorescent diploid must have originated from one singlet haploid A of fluorescence A (e.g. GFP) and one singlet haploid B of fluorescence B (e.g. mRuby2). The mating efficiency is described by the population frequencies of diploids and haploids as given by Eq. 1, here exemplified by haploid A being limiting.

Eq. 1 If haploid $A$ is limiting, then: Mating Efficiency $=($ Diploids $) /\left(\right.$ Haploid A + Diploids) ${ }^{\star} 100$

Statistical and data analysis: Data analysis, statistical analysis and graphing was done in RStudio v1.4.1106 for R (v4.1.2), GraphPad Prism v9.2.0 (GraphPad Software), and FlowLogic ${ }^{T M}$ v8.3 (Inivai Technologies). Sigmoidal dose-response curve fits were computed on nMFls using nonlinear regression by the variable slope (four parameters) model in GraphPad Prism v9.2.0 (GraphPad Software), using default settings (Suppl. Table S9). Significance of

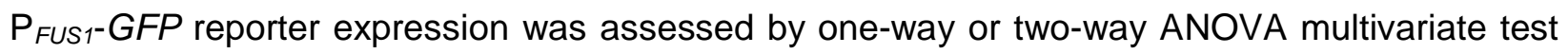
and post-hoc analysis by Dunnett's or Tukey's multiple comparison test, respectively (Suppl. Table S10). Multivariate analysis by determination of Pearson correlation coefficients for SSCA, FSC-A and GFP MFls and simple linear regression was done in GraphPad Prism v9.2.0 (GraphPad Software), using default settings (Suppl. Table S11). Population SSC-A histograms were overlaid and normalized to $100 \%$ using FlowLogic ${ }^{\text {TM }}$ v8.3 (Inivai Technologies). To assess the significance of mating efficiencies, two-way ANOVA multivariate test and post-hoc analysis by Dunnett's multiple comparisons test was done (Suppl. Table S12-14). All statistical tests were done in GraphPad Prism v9.2.0 (GraphPad Software) with a default 95\% confidence interval ( $\square=0.05$ ) applied and multiplicity adjusted $p$-values were reported to account for all multiple comparisons within tests. 
Flow cytometry settings
$B D$
LSRFortessaTM
$X-20$
(BD
Biosciences)
settings

Excitation was done with a blue $488 \mathrm{~nm}$ laser and GFP emission was detected with a 530/30 $\mathrm{nm}$ bandpass (BP) filter (B-530/30) (524V), while FSC was detected with a photodiode detector with a 488/10 nm BP filter (240V) and SSC was detected with a photomultiplier tube (PMT) with a 488/10 nm BP filter (267V).

NovoCyte Quanteon ${ }^{\mathrm{TM}}$ (Agilent) with NovoSampler Q (Agilent) settings: Excitation was done with a blue $488 \mathrm{~nm}$ laser for GFP and a yellow $561 \mathrm{~nm}$ laser for mRuby2, mKate2, FSC, and SSC. GFP emission was detected with a 530/30 nm BP filter (471V), mRuby2 and mKate2 was detected with a 615/20 nm BP filter (616V), while FSC and SSC was detected with a $561 / 14 \mathrm{~nm}$ BP filter (400V). The machine ran with a core diameter of $10.1 \mu \mathrm{m}(24 \mu \mathrm{l} / \mathrm{min}), 2$ mixing cycles every well (1500 RPM, Acc. $=1 \mathrm{~s}$, Dur. $=10 \mathrm{~s}), 1$ rinse cycle every well, and a threshold for event detection at $>150,000$ FSC-H. NovoFlow Solution (Agilent) was used and the machine was calibrated with NovoCyte QC Particles (Agilent).

\section{Procedure for transcriptome analysis of biosensing yeast strains}

Yeast cell harvest and RNA purification: Strains CEN.PK2-1C, CPK2, CPK139, CPK152, and CPK331 were each inoculated in $0.5 \mathrm{ml} \mathrm{SC}$ medium and incubated $\mathrm{O} / \mathrm{N}$ at $30{ }^{\circ} \mathrm{C}$ and 250 RPM. All cultures were then diluted 10 -fold by addition of $4.5 \mathrm{ml} \mathrm{SC}$ medium 20 hrs prior to $\mathrm{OD}_{600}$-adjustment and left for incubation at $30{ }^{\circ} \mathrm{C}$ and $250 \mathrm{RPM}$. Each culture was adjusted to $\mathrm{OD}_{600}=1.0$ the following day and left for additional 2 hrs of incubation before aliquoting $180 \mu \mathrm{l}$ into three wells each in a 96 deep-well plate. $20 \mu \mathrm{l}$ of SC alone, or with 10 -fold increasing concentrations of cognate ligands, and 10\% DMSO was added to each $180 \mu \mathrm{l}$ aliquot and left for shaking incubation at $30{ }^{\circ} \mathrm{C}$ and $250 \mathrm{RPM}$ for $4 \mathrm{hrs}$ prior to harvest. Total RNA was purified using the RNeasy Mini Kit (QIAGEN) according to the protocol. 
Library preparation and sequencing: Purified total RNA was quantified using Qubit fluorometer and qualified using Fragment analyzer. We constructed mRNA libraries using Truseq stranded mRNA kit (illumina) and TruSeq RNA CD indexes (illumina) according to the kit protocols. Libraries were sequenced as paired ends on an Illumina NextSeq 500 at $75 b p$ x 2, with NextSeq High output 150 cycles kit. All raw sequencing data were deposited into the NCBI Short Read Archive under the accession number (PRJNA790752).

Transcriptome analysis: All the raw sequencing reads were trimmed using Trimmomatic (47) to filter sequencing adapters and low-quality reads. The clean reads were aligned against yeast reference genome using STAR (48) with the parameters: “--outFilterMultimapNmax 100 -$\begin{array}{lllllll}\text { alignSJoverhangMin } & 8 & \text {--alignSJDBoverhangMin } & 1 & \text {--outFilterMismatchNmax } & 999 & \text {-- }\end{array}$ alignIntronMax 5000 --alignMatesGapMax 5000 --outSAMtype BAM Unsorted”. The genome reference and gene annotations of Saccharomyces cerevisiae (R64-1-1) were obtained from Ensembl. We used HTSeq-count (49) to calculate raw counts for each yeast gene, with the parameter "-s reverse" to specify the strand information of reads. We applied TMM (trimmed mean of $\mathrm{M}$ values) method (50) implemented in edgeR package to normalize gene expressions. The TMM normalized RPKM (Reads Per Kilobase of transcript, per Million mapped reads) values were further log2 transformed to generate heatmap and other plots using pheatmap (51).

DEG analysis: Genes with cpm (counts per million mapped reads) above 1 in at least three samples were kept for the DEG analysis (52), which was performed by the "exactTest" function in edgeR between each pair of conditions (53). We identified DEGs in each pairwise analysis using the standard: log2-transformed fold change above 1 and FDR under 0.05 . We applied TopGO (54) to perform GO enrichment analysis. Benjamin-Hochberg false discovery rate correction was used to adjust $P$ values to determine the significant enriched $G O$ terms. 


\section{Figure}

\section{legends}

\section{Figure 1. Biosensing yeast characterization and transcriptome analysis.}

A. Sensitization of Saccharomyces cerevisiae (S. cerevisiae) for biosensing with heterologous G protein-coupled receptor (hGPCR)-signaling via its mating pathway. $S$. cerevisiae strains are genetic mutants for sst2 $\Delta$ to diminish inhibition of signal transduction mediated by $\mathrm{G}_{\beta \gamma}$ subunits. $\mathrm{G}_{\alpha}$ expression tuning controls background and ligand-activated signal transduction schematized as fold-change of GFP expressed from the $P_{F U S 1}-$ GFP reporter ("Reporter assay"). The yeast shmoo phenotype ("Morphology"), following from mating pathway activation, can be observed as increased forward-scatter (FSC) and side-scatter (SSC) with flow cytometry. B. Normalized median fluorescence intensities (nMFI, left) and MFI fold-changes (right) for biosensing yeast strains expressing A2bR, $\mathrm{MT}_{1}, 5-\mathrm{HT}_{4 \mathrm{~b}}$, or Mam2 (CPK153-161, and CPK165-167) are shown for low $\left(P_{R N R 2}\right)$, medium $\left(P_{A L D 6}\right)$, and strong $\left(P_{P G K 1}\right) G_{\alpha}$ subunit expression with or without cognate ligands in dosage range $0-100 \mu \mathrm{M}$ shown as $\log [\mathrm{M}]$. Parental strains without GPCRs or ligand supplementation (CPK131-136) are shown as red circles (left). nMFI is normalized for all strains to the $P_{P G K 1}-G_{\alpha}$ strain without ligand supplementation within each hGPCR design (left), and foldchange is determined relative to the background with no supplementation for each individual strain. Data represent means and standard deviations from three biological replicates, and curves were fitted using GraphPad Prism variable slope (four parameter) nonlinear regression (see Methods). C. Venn diagram illustrating transcriptome analysis results by depicting overlapping and unique differentially expressed genes (DEGs) between biosensing yeast strains expressing either A2bR (CPK331), $\mathrm{MT}_{1}$ (CPK139), or 5-HT $4 \mathrm{~b}$ (CPK152), vs wild-type (CEN.PK21C, WT) and sst2 $\Delta$ sensitized yeast (CPK2) across a gradient of cognate ligand concentrations $(0-100 \mu \mathrm{M})$. D. Heat map showing relative expression for 57 DEGs identified from transcriptome analysis that overlap between all strains and conditions.

Figure 2. One single genetic edit enables GPCR-signaling in probiotic yeast. 
A. Homozygous mating-type switch in MATa/a diploid cells with CRISPR/Cas9 (left). Cas9 is pre-expressed in $S$. boulardii prior to transformation with a single gRNA plasmid that targets MATa in the genome for double-strand break (DSB). The endogenous MATa copy templates Cas9-induced double-strand break repair (not shown) and converts the cell into a MATa/a diploid. $\mathrm{G}_{\alpha}$ expression is engineered from the PGK1 promoter, and heterologous GPCRs (hGPCRs) are expressed from $2 \mu$ plasmids (right). B-E. Median fluorescence intensities (MFI)

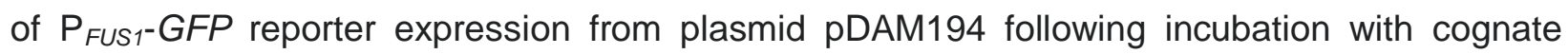
ligands in dosages 0-100 $\mu \mathrm{M}$. B. SB14 (MATa/a, left) and SB17 (MATa/a, right) were incubated with yeast pheromone (a-factor). C-E. Cognate ligand sensing in hGPCR-signaling MATa/a cells for C. A2bR sensing adenosine (SB48), D. $M T_{1}$ sensing melatonin (SB49), and E. Mam2 sensing P-factor (SB50). Means and standard deviations represent three biological replicates. Statistical significance was determined using two-way analysis of variance (ANOVA) with Tukey's multiple comparison test $(\mathbf{B})$ or one-way ANOVA with Dunnett's multiple comparison test in GraphPad Prism ( $\left.{ }^{* *} p \leq 0.01\right)$ (C-E) (see Methods).

Figure 3. Mating pathway activation and changed morphology correlate.

A-C. Increased side-scatter area (SSC-A) correlates with median fluorescence intensities (MFI)

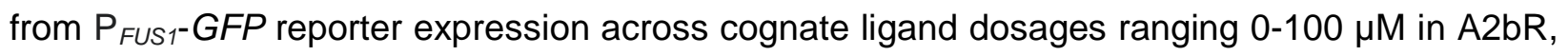
$\mathrm{MT}_{1}, 5-\mathrm{HT}_{4 \mathrm{~b}}$, and Mam2 biosensing strains of $S$. cerevisiae (CPK153-161 and CPK165-167). Linear regression was performed using Graphpad Prism, and $\mathrm{R}^{2}$ is reported for each analysis (see Methods). A. All data $(n=216)$. B. Highlights and correlation of each $\mathrm{G}_{\alpha}$ expression design in all data $(n=72 \times 3)$. C. Individual representation of correlation for all hGPCR designs $(n=54$ $x$ 4). D. Representative morphologies in $S$. cerevisiae strains from panels $A-C$ in absence (ligand) or presence of cognate ligands (+ligand, $100 \mu \mathrm{M}$ ) for $\mathrm{A} 2 \mathrm{bR}, \mathrm{MT}_{1}$, and 5-HT $4 \mathrm{~b}$, or Mam2 $(1 \mu \mathrm{M})$. Size bars illustrate $10 \mu \mathrm{m}$. E. Increasing SSC-A for each strain design in panels A-C 
across $0-100 \mu \mathrm{M}$ cognate ligand supplementation as indicated. SSC-A is normalized for all strains to the $P_{P G K 1}-G_{\alpha}$ strain without ligand supplementation within each hGPCR design.

\section{Figure 4. Heterologous GPCRs support semi-synthetic yeast mating.}

A. Schematic outline showing production, supplementation, and sensing of melatonin $\left(\mathrm{MT}_{1}\right)$, serotonin $\left(5-\mathrm{HT}_{4 \mathrm{~b}}\right)$, or P-factor (Mam2) in yeast mating pairs. Adenosine (A2bR) was present solely from supplementation. B-E. Percentage of mating efficiencies (second-axis) and diploid frequencies (third-axis) at increasing cognate ligand supplementation dosages (first-axis, 0-100 $\mu \mathrm{M}$ shown as $\log [\mathrm{M}])$ were scored following $5 \mathrm{hrs}$ co-incubation at $30^{\circ} \mathrm{C}$ and $250 \mathrm{RPM}$ in a 96 deep-well plate. Results are presented as means with standard deviations determined from five biological replicates. CPK46xSBY55 and CPK121xSBY55 or CPK124xSBY55 were used as references for positive and negative mating pair controls with relevant ligand supplementation, respectively. No ligand supplementation controls are indicated (-). B. CPK331xSBY55 mating at different supplementation doses of adenosine. C. CPK142xSBY55 (no production) and CPK142xSBY155 (P-factor production) mating crosses were investigated with and without additional supplementation of P-factor in 1\% DMSO in indicated concentrations. D. CPK139xSBY55 (no production), CPK139xSBY92 (low melatonin production), and CPK139xSBY139 (high melatonin production) mating crosses were investigated with and without additional supplementation of melatonin in indicated concentrations. E. CPK152xSBY55 (no production), CPK152xSBY91 (low serotonin production), and CPK152xSBY138 (high serotonin production) mating crosses were investigated with and without additional supplementation of serotonin in indicated concentrations. Statistical significance was determined for mating efficiencies using two-way analysis of variance (ANOVA) with Dunnett's multiple comparisons test, relative to the condition of no production and no supplementation for each setup, using GraphPad Prism ( ${ }^{*} p \leq 0.05,{ }^{* *} p \leq 0.01$ ) (see Methods). 
Figure 5. Heterologous GPCRs support full synthetic and autonomous yeast mating.

A. Individual or combined synthetic pheromone (cognate ligand) supplementation and biosensing of adenosine $(\mathrm{A} 2 \mathrm{bR})$, melatonin $\left(\mathrm{MT}_{1}\right)$, and serotonin $\left(5-\mathrm{HT}_{4 \mathrm{~b}}\right)$ in yeast mating pairs. B-D. Percentage of mating efficiencies (second-axis) and diploid frequencies (third-axis) following supplementation of one or both synthetic pheromones at increasing dosages (firstaxis, 0-100 $\mu \mathrm{M}$ shown as $\log [\mathrm{M}])$ were scored following $5 \mathrm{hrs}$ co-incubation at $30{ }^{\circ} \mathrm{C}$ and 250 RPM in a 96 deep-well plate. Results are presented as means with standard deviations determined from five biological replicates. Negative controls without ligand supplementation are indicated (-). Mating crosses were done with B. adenosine or melatonin supplementation alone or in combination for CPK139xSBY157, C. adenosine or serotonin alone or in combination for CPK152xSBY157, and D. melatonin or serotonin alone or in combination for CPK152xSBY156. E. Paracrine signaling from serotonin and P-factor production in an autonomous yeast mating pair. Autonomous mating between strains sending and receiving P-factor and serotonin: CPK152xSBY173 (no production), CPK152xSBY175 (production of serotonin only), CPK448xSBY173 (production of P-factor only), CPK448xSBY175 (production of both serotonin and P-factor). CPK124xSBY172 (No GPCR) without hGPCRs (Mam2 and 5-HT $4 \mathrm{~b}$ ) was included as negative control. Means and standard deviations represent five biological replicates. Statistical significance was determined for mating efficiencies using one-way (B-D) and two-way (E) analysis of variance (ANOVA) with Dunnett's multiple comparisons test, relative to the negative control in each setup, using GraphPad Prism ( $\left.{ }^{* *} p \leq 0.01\right)$ (see Methods).

\section{References}

1. $\varnothing$. Winge, On haplophase and diplophase in some Saccharomycetes (Hagerup in Komm., 1935). 
2. C. C. Lindegren, G. Lindegren, A New Method for Hybridizing Yeast. Proc. Natl. Acad. Sci. U. S. A. 29, 306-308 (1943).

3. L. H. Hartwell, Mutants of Saccharomyces cerevisiae unresponsive to cell division control by polypeptide mating hormone. J. Cell Biol. 85, 811-822 (1980).

4. S. L. Forsburg, The art and design of genetic screens: yeast. Nat. Rev. Genet. 2, 659-668 (2001).

5. K. King, H. G. Dohlman, J. Thorner, M. G. Caron, R. J. Lefkowitz, Control of yeast mating signal transduction by a mammalian beta 2-adrenergic receptor and Gs alpha subunit. Science. 250, 121-123 (1990).

6. A. J. Brown, S. L. Dyos, M. S. Whiteway, J. H. White, M. A. Watson, M. Marzioch, J. J. Clare, D. J. Cousens, C. Paddon, C. Plumpton, M. A. Romanos, S. J. Dowell, Functional coupling of mammalian receptors to the yeast mating pathway using novel yeast/mammalian G protein alpha-subunit chimeras. Yeast. 16, 11-22 (2000).

7. S. Billerbeck, J. Brisbois, N. Agmon, M. Jimenez, J. Temple, M. Shen, J. D. Boeke, V. W. Cornish, A scalable peptide-GPCR language for engineering multicellular communication. Nat. Commun. 9, 5057 (2018).

8. W. M. Shaw, H. Yamauchi, J. Mead, G.-O. F. Gowers, D. J. Bell, D. Öling, N. Larsson, M. Wigglesworth, G. Ladds, T. Ellis, Engineering a Model Cell for Rational Tuning of GPCR Signaling. Cell. 177, 782-796.e27 (2019).

9. J. E. Haber, Mating-type gene switching in Saccharomyces cerevisiae. Annu. Rev. Genet. 32, 561-599 (1998).

10. B. Lengger, M. K. Jensen, Engineering G protein-coupled receptor signalling in yeast for biotechnological and medical purposes. FEMS Yeast Res. 20 (2020), doi:10.1093/femsyr/foz087.

11. N. J. Kapolka, G. J. Taghon, J. B. Rowe, W. M. Morgan, J. F. Enten, N. A. Lambert, D. G. Isom, DCyFIR: a high-throughput CRISPR platform for multiplexed G protein-coupled receptor profiling and ligand discovery. Proc. Natl. Acad. Sci. U. S. A. 117, 13117-13126 (2020).

12. N. Ostrov, M. Jimenez, S. Billerbeck, J. Brisbois, J. Matragrano, A. Ager, V. W. Cornish, A modular yeast biosensor for low-cost point-of-care pathogen detection. Sci Adv. 3, e1603221 (2017).

13. B. M. Scott, C. Gutiérrez-Vázquez, L. M. Sanmarco, J. A. da Silva Pereira, Z. Li, A. Plasencia, P. Hewson, L. M. Cox, M. O’Brien, S. K. Chen, P. M. Moraes-Vieira, B. S. W. Chang, S. G. Peisajovich, F. J. Quintana, Self-tunable engineered yeast probiotics for the treatment of inflammatory bowel disease. Nat. Med. 27, 1212-1222 (2021).

14. C. J. Roberts, B. Nelson, M. J. Marton, R. Stoughton, M. R. Meyer, H. A. Bennett, Y. D. He, H. Dai, W. L. Walker, T. R. Hughes, M. Tyers, C. Boone, S. H. Friend, Signaling and circuitry of multiple MAPK pathways revealed by a matrix of global gene expression profiles. Science. 287, 873-880 (2000). 
15. V. L. MacKay, X. Li, M. R. Flory, E. Turcott, G. L. Law, K. A. Serikawa, X. L. Xu, H. Lee, D. R. Goodlett, R. Aebersold, L. P. Zhao, D. R. Morris, Gene expression analyzed by highresolution state array analysis and quantitative proteomics: response of yeast to mating pheromone. Mol. Cell. Proteomics. 3, 478-489 (2004).

16. M. Leutert, R. A. Rodríguez-Mias, N. K. Fukuda, J. Villén, R2-P2 rapid-robotic phosphoproteomics enables multidimensional cell signaling studies. Mol. Syst. Biol. 15, e9021 (2019).

17. A. M. Janiak, H. Sargsyan, J. Russo, F. Naider, M. Hauser, J. M. Becker, Functional expression of the Candida albicans a-factor receptor in Saccharomyces cerevisiae. Fungal Genet. Biol. 42, 328-338 (2005).

18. J. Gonçalves-Sá, A. Murray, Asymmetry in sexual pheromones is not required for ascomycete mating. Curr. Biol. 21, 1337-1346 (2011).

19. T. J. Fowler, S. M. DeSimone, M. F. Mitton, J. Kurjan, C. A. Raper, Multiple sex pheromones and receptors of a mushroom-producing fungus elicit mating in yeast. $\mathrm{Mol}$. Biol. Cell. 10, 2559-2572 (1999).

20. H. G. Dohlman, J. Thorner, RGS proteins and signaling by heterotrimeric G proteins. J. Biol. Chem. 272, 3871-3874 (1997).

21. B. Lengger, E. E. Hoch-Schneider, C. N. Jensen, T. Jakočiūnas, E. D. Jensen, M. K. Jensen, Serotonin GPCR-based biosensing modalities in yeast. bioRxiv (2021), p. 2021.07.12.452006.

22. W.-B. Zhang, J.-M. Navenot, B. Haribabu, H. Tamamura, K. Hiramatu, A. Omagari, G. Pei, J. P. Manfredi, N. Fujii, J. R. Broach, S. C. Peiper, A point mutation that confers constitutive activity to CXCR4 reveals that T140 is an inverse agonist and that AMD3100 and ALX404C are weak partial agonists. J. Biol. Chem. 277, 24515-24521 (2002).

23. C. Weston, D. Poyner, V. Patel, S. Dowell, G. Ladds, Investigating G protein signalling bias at the glucagon-like peptide-1 receptor in yeast. Br. J. Pharmacol. 171, 3651-3665 (2014).

24. P. T. Spellman, G. Sherlock, M. Q. Zhang, V. R. lyer, K. Anders, M. B. Eisen, P. O. Brown, D. Botstein, B. Futcher, Comprehensive Identification of Cell Cycle-regulated Genes of the Yeast Saccharomyces cerevisiae by Microarray Hybridization. MBoC. 9, 3273-3297 (1998).

25. L. A. Price, E. M. Kajkowski, J. R. Hadcock, B. A. Ozenberger, M. H. Pausch, Functional coupling of a mammalian somatostatin receptor to the yeast pheromone response pathway. Mol. Cell. Biol. 15, 6188-6195 (1995).

26. T. Kelesidis, C. Pothoulakis, Efficacy and safety of the probiotic Saccharomyces boulardii for the prevention and therapy of gastrointestinal disorders. Therap. Adv. Gastroenterol. 5, 111-125 (2012).

27. L. C. Edwards-Ingram, M. E. Gent, D. C. Hoyle, A. Hayes, L. I. Stateva, S. G. Oliver, Comparative genomic hybridization provides new insights into the molecular taxonomy of the Saccharomyces sensu stricto complex. Genome Res. 14, 1043-1051 (2004). 
28. B. Offei, P. Vandecruys, S. De Graeve, M. R. Foulquié-Moreno, J. M. Thevelein, Unique genetic basis of the distinct antibiotic potency of high acetic acid production in the probiotic yeast Saccharomyces cerevisiae var. boulardii. Genome Res. 29, 1478-1494 (2019).

29. H. Roman, S. M. Sands, Heterogeneity of Clones of Saccharomyces Derived from Haploid Ascospores. Proc. Natl. Acad. Sci. U. S. A. 39, 171-179 (1953).

30. J. E. DiCarlo, J. E. Norville, P. Mali, X. Rios, J. Aach, G. M. Church, Genome engineering in Saccharomyces cerevisiae using CRISPR-Cas systems. Nucleic Acids Res. 41, 4336-4343 (2013).

31. T. I. Moore, C.-S. Chou, Q. Nie, N. L. Jeon, T.-M. Yi, Robust spatial sensing of mating pheromone gradients by yeast cells. PLoS One. 3, e3865 (2008).

32. C.-S. Chou, T. I. Moore, S. D. Chang, Q. Nie, T.-M. Yi, Signaling regulated endocytosis and exocytosis lead to mating pheromone concentration dependent morphologies in yeast. FEBS Lett. 586, 4208-4214 (2012).

33. S. Hennig, A. Clemens, G. Rödel, K. Ostermann, A yeast pheromone-based inter-species communication system. Appl. Microbiol. Biotechnol. 99, 1299-1308 (2015).

34. L. B. Huberman, A. W. Murray, Genetically engineered transvestites reveal novel mating genes in budding yeast. Genetics. 195, 1277-1290 (2013).

35. N. Barkai, M. D. Rose, N. S. Wingreen, Protease helps yeast find mating partners. Nature. 396, 422-423 (1998).

36. D. Durmusoglu, I. S. Al'Abri, S. P. Collins, J. Cheng, A. Eroglu, C. L. Beisel, N. Crook, In Situ Biomanufacturing of Small Molecules in the Mammalian Gut by Probiotic Saccharomyces boulardii. ACS Synth. Biol. 10, 1039-1052 (2021).

37. J. Bitinaite, M. Rubino, K. H. Varma, I. Schildkraut, R. Vaisvila, R. Vaiskunaite, USER ${ }^{\text {TM }}$ friendly DNA engineering and cloning method by uracil excision. Nucleic Acids Res. 35, 1992-2002 (2007).

38. M. M. Jessop-Fabre, T. Jakočiūnas, V. Stovicek, Z. Dai, M. K. Jensen, J. D. Keasling, I. Borodina, EasyClone-MarkerFree: A vector toolkit for marker-less integration of genes into Saccharomyces cerevisiae via CRISPR-Cas9. Biotechnol. J. 11, 1110-1117 (2016).

39. E. D. Jensen, M. Laloux, B. J. Lehka, L. E. Pedersen, T. Jakočiūnas, M. K. Jensen, J. D. Keasling, A synthetic RNA-mediated evolution system in yeast. Nucleic Acids Res. 49, e88 (2021).

40. T. Jakočiūnas, I. Bonde, M. Herrgård, S. J. Harrison, M. Kristensen, L. E. Pedersen, M. K. Jensen, J. D. Keasling, Multiplex metabolic pathway engineering using CRISPR/Cas9 in Saccharomyces cerevisiae. Metab. Eng. 28, 213-222 (2015).

41. M. E. Lee, W. C. DeLoache, B. Cervantes, J. E. Dueber, A Highly Characterized Yeast Toolkit for Modular, Multipart Assembly. ACS Synth. Biol. 4, 975-986 (2015).

42. Z.-X. Wang, J. R. Broach, S. C. Peiper, in Transmembrane Signaling Protocols, H. Ali, B. Haribabu, Eds. (Humana Press, Totowa, NJ, 2006), pp. 115-127. 
43. S. M. Germann, S. A. Baallal Jacobsen, K. Schneider, S. J. Harrison, N. B. Jensen, X. Chen, S. G. Stahlhut, I. Borodina, H. Luo, J. Zhu, J. Maury, J. Forster, Glucose-based microbial production of the hormone melatonin in yeast Saccharomyces cerevisiae.

Biotechnol. J. 11, 717-724 (2016).

44. D. Romero-Suarez, T. Wulff, Y. Rong, T. Jakočiu Nas, S. Yuzawa, J. D. Keasling, M. K. Jensen, A Reporter System for Cytosolic Protein Aggregates in Yeast. ACS Synth. Biol. 10, 466-477 (2021).

45. E. D. Jensen, R. Ferreira, T. Jakočiūnas, D. Arsovska, J. Zhang, L. Ding, J. D. Smith, F. David, J. Nielsen, M. K. Jensen, J. D. Keasling, Transcriptional reprogramming in yeast using dCas9 and combinatorial gRNA strategies. Microb. Cell Fact. 16, 46 (2017).

46. I. Sadowska-Bartosz, A. Pączka, M. Mołoń, G. Bartosz, Dimethyl sulfoxide induces oxidative stress in the yeast Saccharomyces cerevisiae. FEMS Yeast Res. 13, 820-830 (2013).

47. A. M. Bolger, M. Lohse, B. Usadel, Trimmomatic: a flexible trimmer for Illumina sequence data. Bioinformatics. 30, 2114-2120 (2014).

48. A. Dobin, C. A. Davis, F. Schlesinger, J. Drenkow, C. Zaleski, S. Jha, P. Batut, M. Chaisson, T. R. Gingeras, STAR: ultrafast universal RNA-seq aligner. Bioinformatics. 29, 15-21 (2013).

49. S. Anders, P. T. Pyl, W. Huber, HTSeq--a Python framework to work with high-throughput sequencing data. Bioinformatics. 31, 166-169 (2015).

50. M. D. Robinson, A. Oshlack, A scaling normalization method for differential expression analysis of RNA-seq data. Genome Biol. 11, R25 (2010).

51. R. Kolde, pheatmap: Pretty heatmaps. The Comprehensive R Archive Network (2015).

52. S. Anders, D. J. McCarthy, Y. Chen, M. Okoniewski, G. K. Smyth, W. Huber, M. D. Robinson, Count-based differential expression analysis of RNA sequencing data using $R$ and Bioconductor. Nat. Protoc. 8, 1765-1786 (2013).

53. M. D. Robinson, D. J. McCarthy, G. K. Smyth, edgeR: a Bioconductor package for differential expression analysis of digital gene expression data. Bioinformatics. 26, 139-140 (2010).

54. A. A. J. Rahnenfuhrer. topGO: Enrichment Analysis for Gene Ontology. R package version 2.46 .0 (2021). 
bioRxiv preprint doi: https://doi.org/10.1101/2022.01.11.475777; this version posted January 12,2022 . The copyright holder for this preprint (which was not certified by peer review) is the author/funder, who has granted bioRxiv a license to display the preprint in perpetuity. It is made available under aCC-BY-ND 4.0 International license.

A

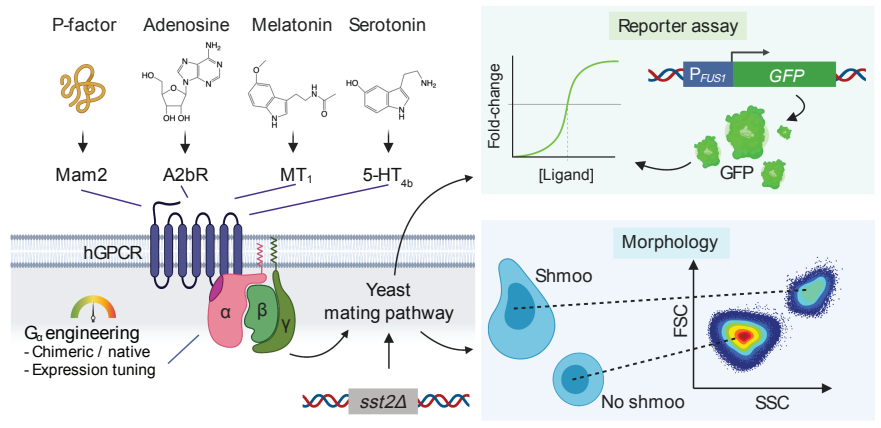

B
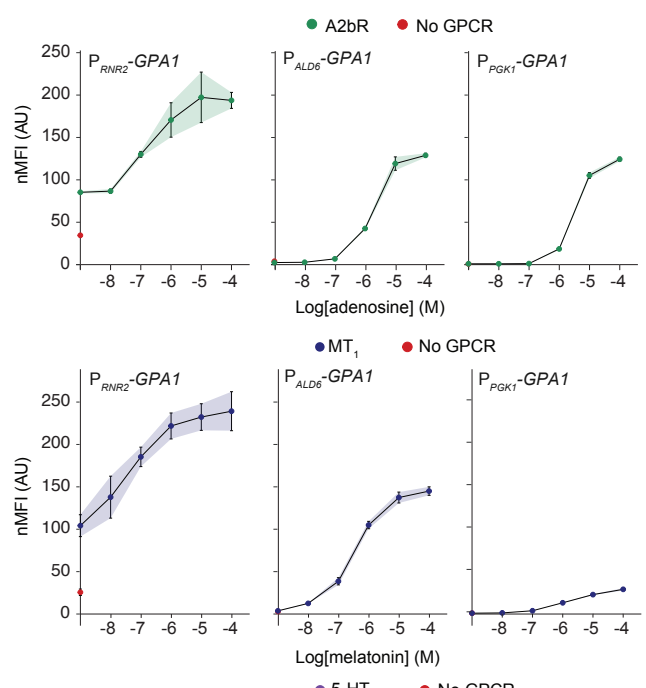

- 5-HT $\quad$ - No GPCR

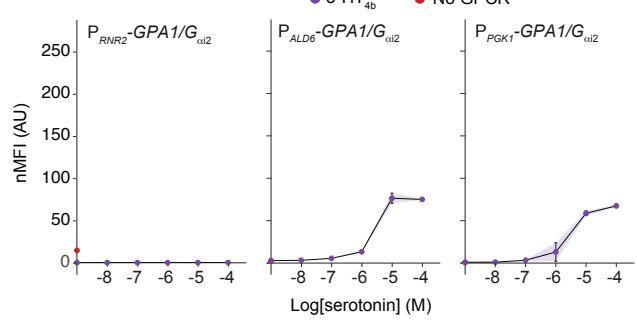

- Mam2 - No GPCR

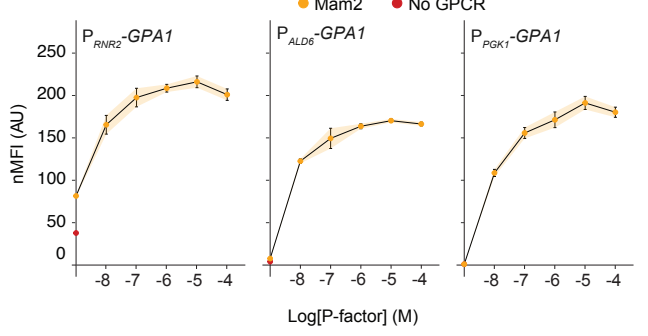

C
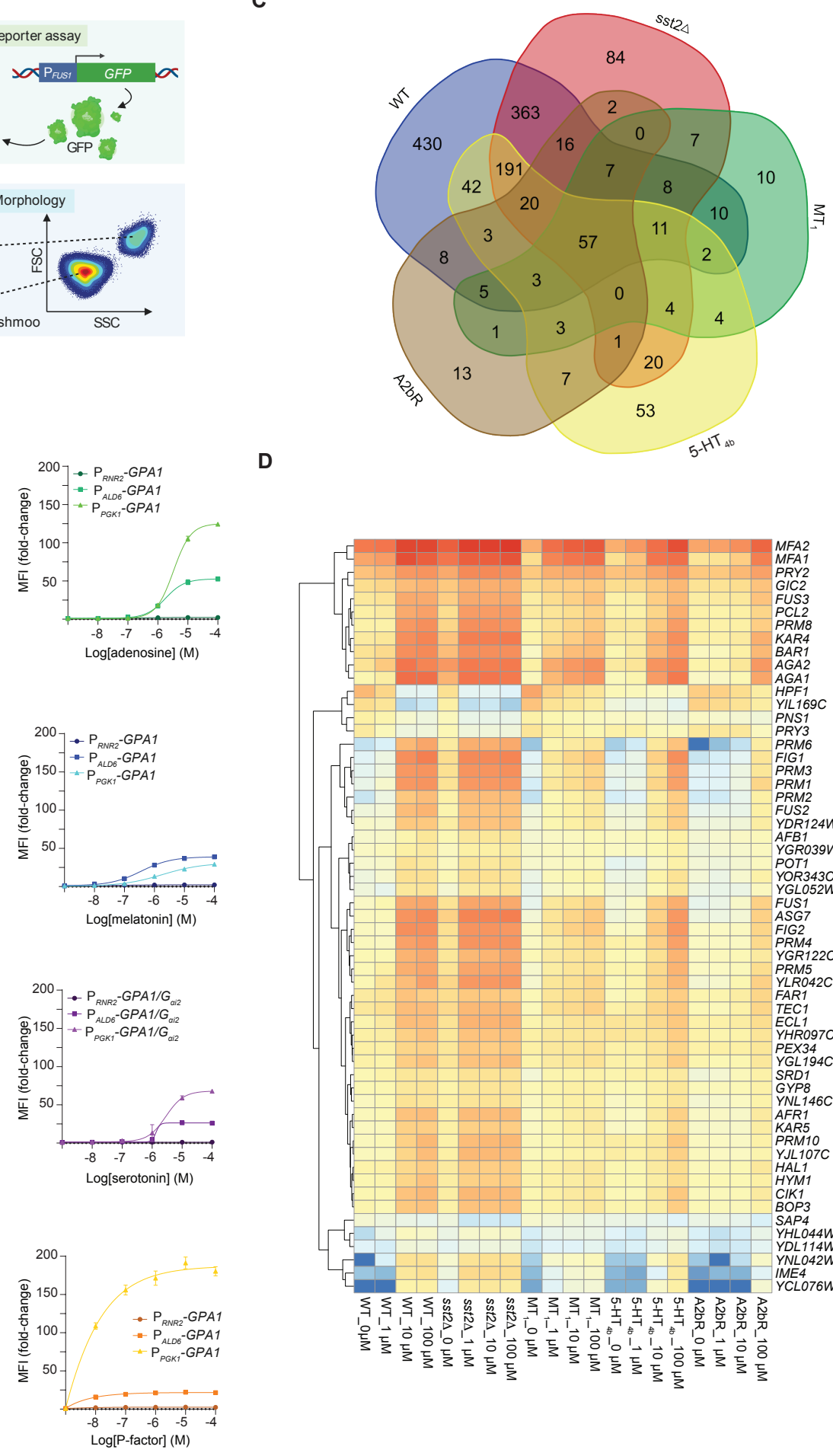

Figure 1.

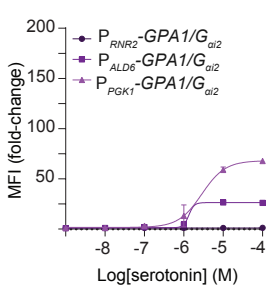

D
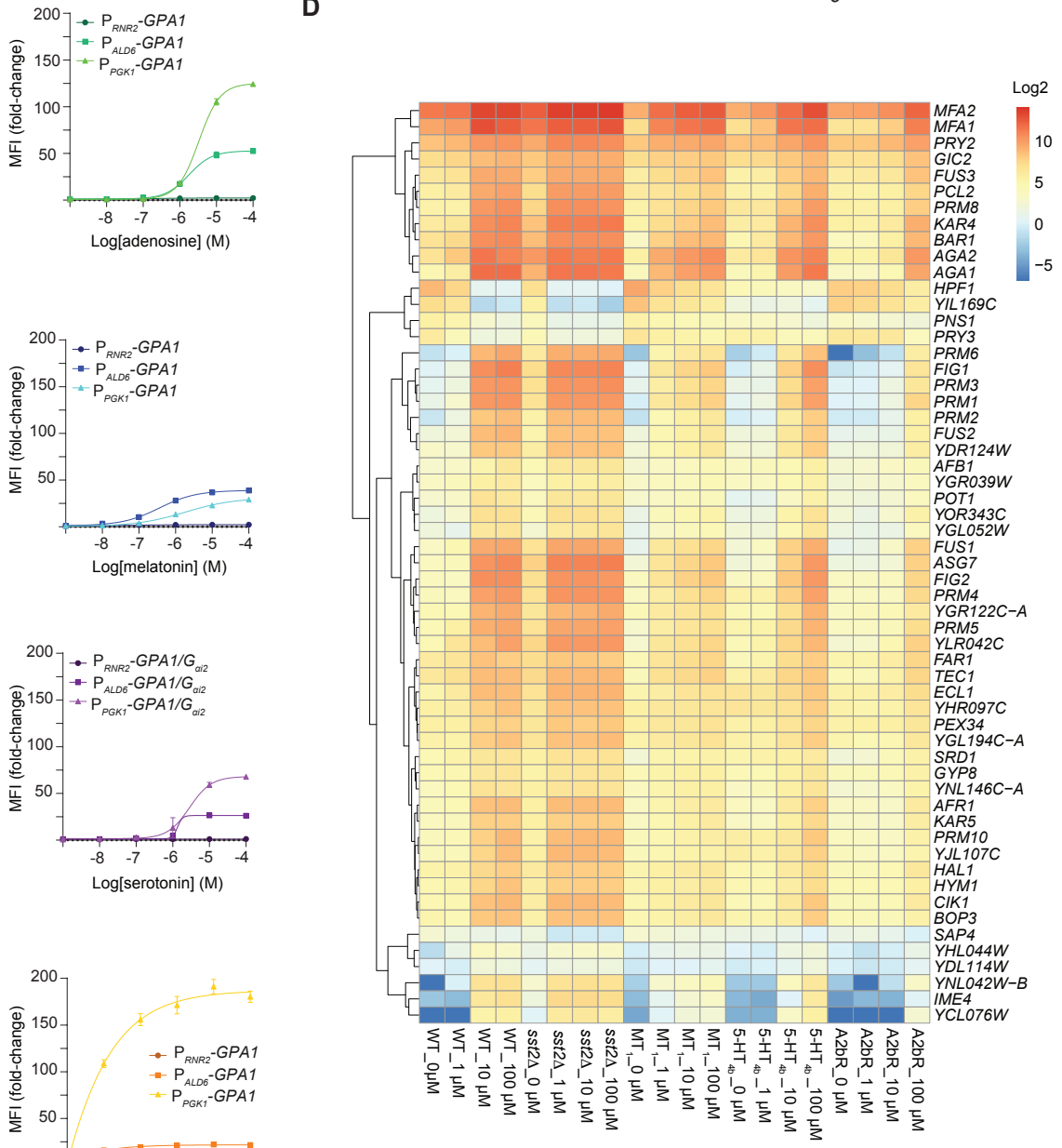
bioRxiv preprint doi: https://doi.org/10.1101/2022.01.11.475777; this version posted January 12, 2022. The copyright holder for this preprint (which was not certified by peer review) is the author/funder, who has granted bioRxiv a license to display the preprint in perpetuity. It is made available under aCC-BY-ND 4.0 International license.

A

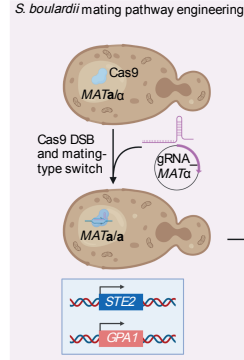

C

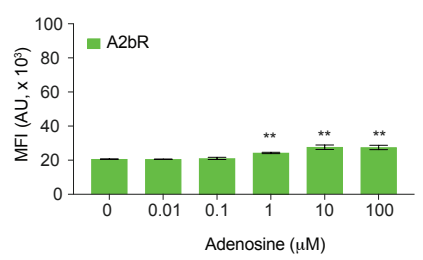

hGPCR expression in $S$. boulardii

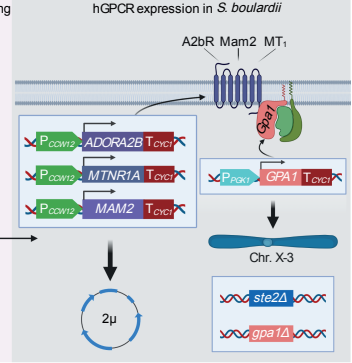

D
B

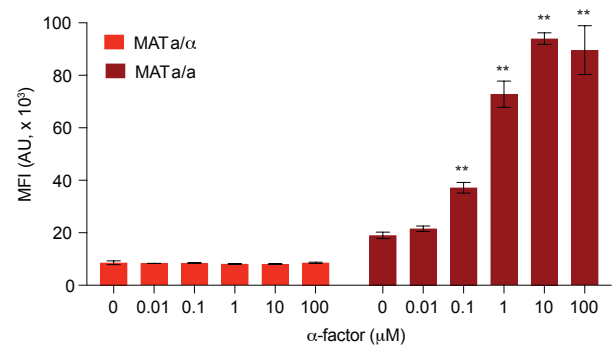

E
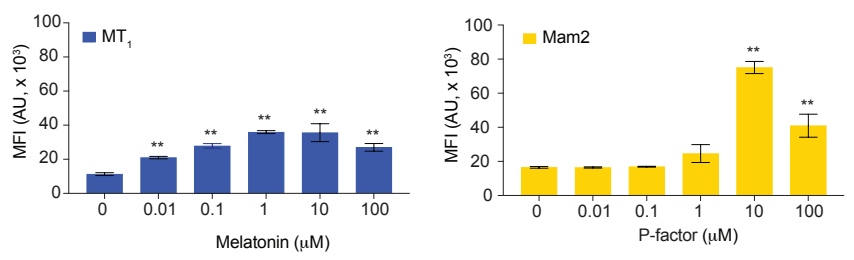

Figure 2. 
bioRxiv preprint doi: https://doi.org/10.1101/2022.01.11.475777; this version posted January 12,2022 . The copyright holder for this preprint (which was not certified by peer review) is the author/funder, who has granted bioRxiv a license to display the preprint in perpetuity. It is made available under aCC-BY-ND 4.0 International license.

A

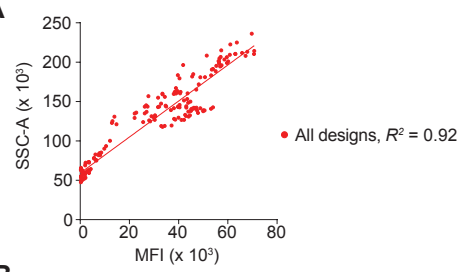

B

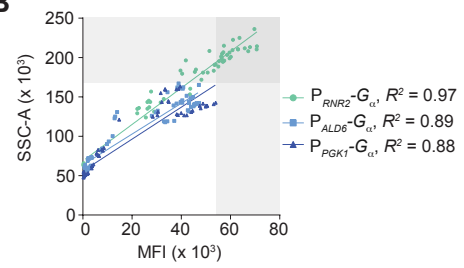

D

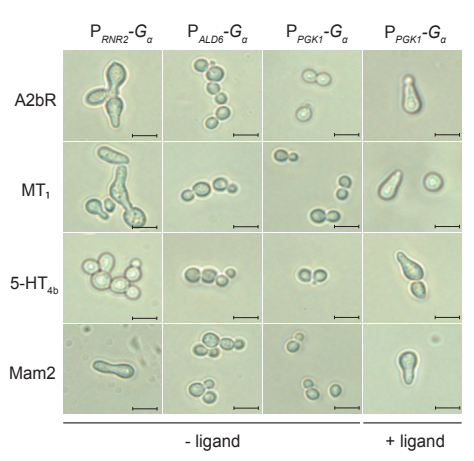

C
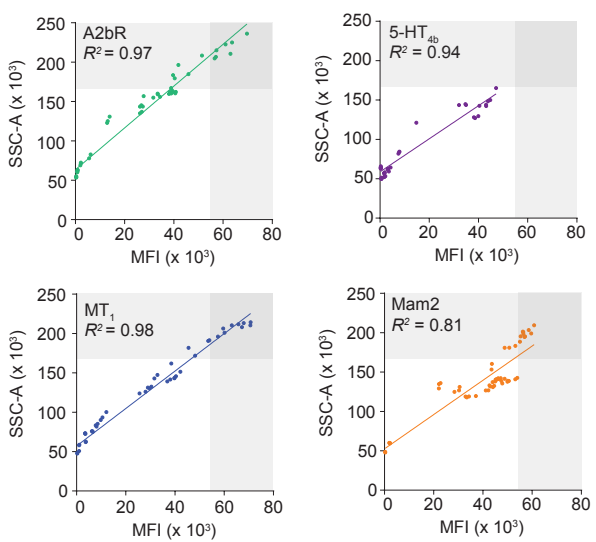

E
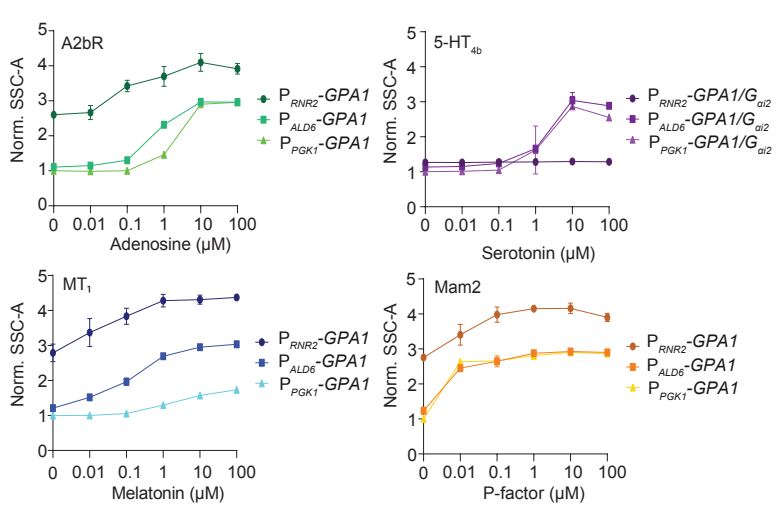

Serotonin $(\mu \mathrm{M})$

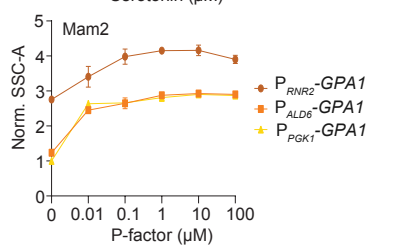

Figure 3. 
bioRxiv preprint doi: https://doi.org/10.1101/2022.01.11.475777; this version posted January 12,2022 . The copyright holder for this preprint (which was not certified by peer review) is the author/funder, who has granted bioRxiv a license to display the preprint in perpetuity. It is made available under aCC-BY-ND 4.0 International license.

A
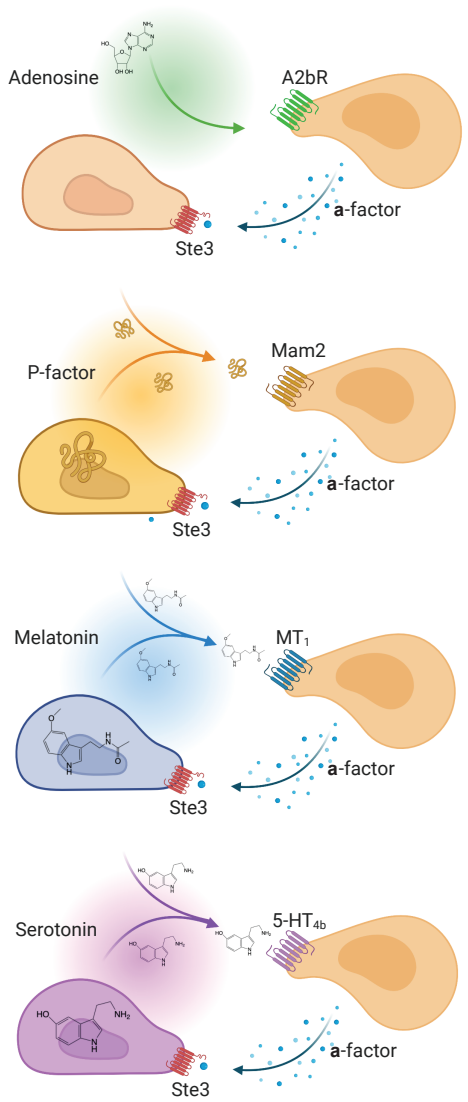

B

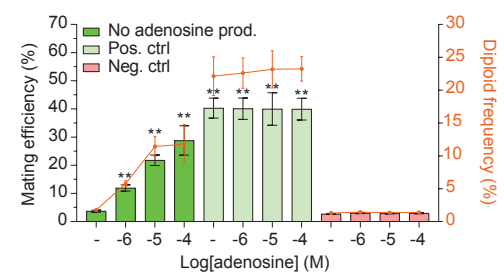

C

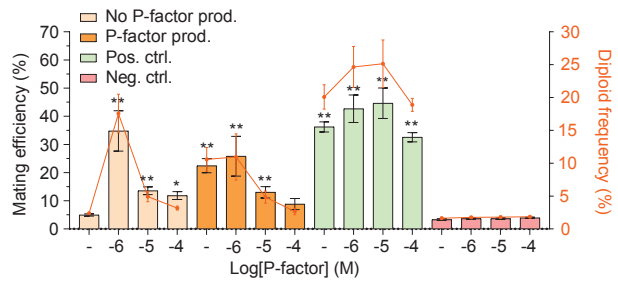

D

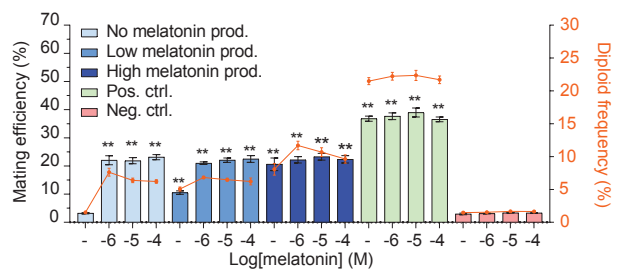

E

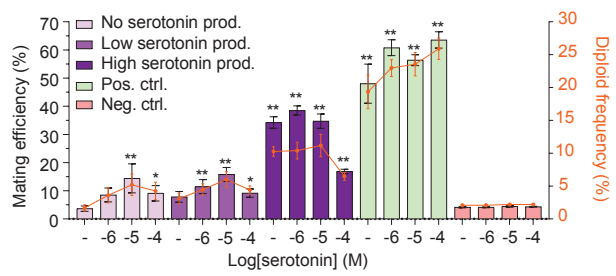

Figure 4. 
bioRxiv preprint doi: https://doi.org/10.1101/2022.01.11.475777; this version posted January 12,2022 . The copyright holder for this preprint (which was not certified by peer review) is the author/funder, who has granted bioRxiv a license to display the preprint in perpetuity. It is made available under aCC-BY-ND 4.0 International license.

A

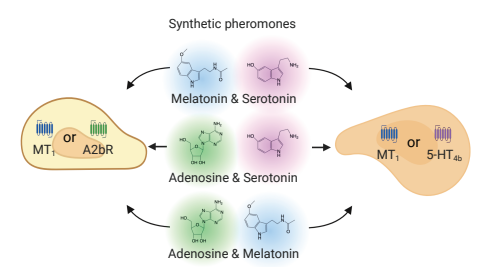

B

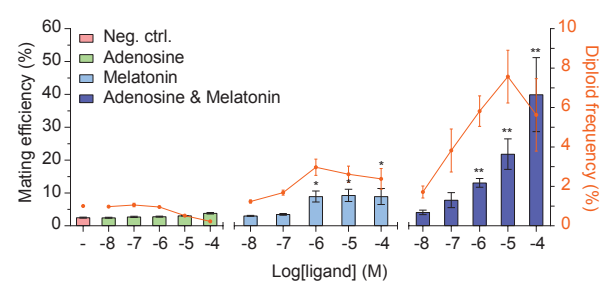

C

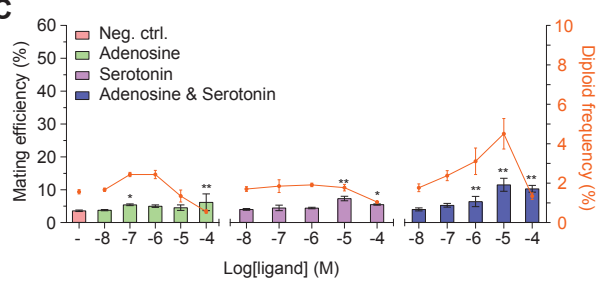

$\mathbf{E}$

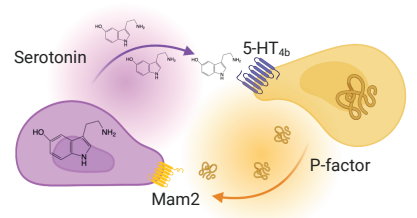

D ${ }^{60} 7$ 口 Neg. ctrl.
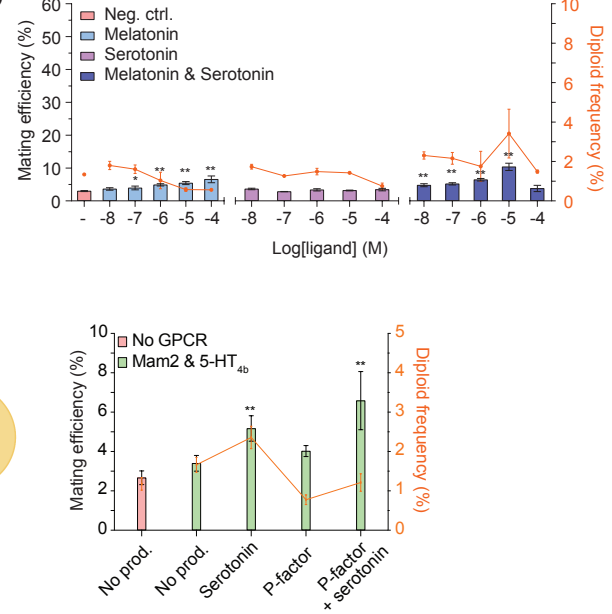

Figure 5. 